\title{
COMPOSITE GENERALIZED LAGUERRE-LEGENDRE SPECTRAL METHOD WITH DOMAIN DECOMPOSITION AND ITS APPLICATION TO FOKKER-PLANCK EQUATION IN AN INFINITE CHANNEL
}

\author{
BEN-YU GUO AND TIAN-JUN WANG
}

\begin{abstract}
In this paper, we propose a composite generalized LaguerreLegendre spectral method for partial differential equations on two-dimensional unbounded domains, which are not of standard types. Some approximation results are established, which are the mixed generalized Laguerre-Legendre approximations coupled with domain decomposition. These results play an important role in the related spectral methods. As an important application, the composite spectral scheme with domain decomposition is provided for the Fokker-Planck equation in an infinite channel. The convergence of the proposed scheme is proved. An efficient algorithm is described. Numerical results show the spectral accuracy in the space of this approach and coincide well with theoretical analysis. The approximation results and techniques developed in this paper are applicable to many other problems on unbounded domains. In particular, some quasi-orthogonal approximations are very appropriate for solving PDEs, which behave like parabolic equations in some directions, and behave like hyperbolic equations in other directions. They are also useful for various spectral methods with domain decompositions, and numerical simulations of exterior problems.
\end{abstract}

\section{InTRODUCTION}

The spectral method has developed rapidly in the past three decades. Its main merit is its high accuracy. Along with extensive applications of Legendre and Chebyshev spectral methods for bounded domains (cf. [1, 2, 6, 9, 11, 12]), considerable progress has been made recently in spectral methods for unbounded domains. Among these methods, a direct and commonly used approach is based on certain orthogonal approximations on infinite intervals, i.e., the Hermite and Laguerre spectral methods ( see, e.g., [5, 10, 13, 16, 18, 23, 24, 28, 32]). Some authors also developed composite Laguerre-Legendre spectral methods for the half line and mixed Laguerre-Legendre spectral methods for an infinite strip; see [9, 15, 21, 31.

Received by the editor April 13, 2007 and, in revised form, February 9, 2008.

2000 Mathematics Subject Classification. Primary 65M70, 41A30, 82C99.

Key words and phrases. Composite generalized Laguerre-Legendre spectral method, quasiorthogonal approximation, domain decomposition, Fokker-Planck equation in an infinite channel.

The work of the first author was supported in part by The Grant of Science and Technology Commission of Shanghai Municipality N.75105118, The Shanghai Leading Academic Discipline Project N.T0401, and The Fund for E-institutes of Shanghai Universities N.E03004.

The work of the second author was supported in part by The Doctor Fund of Henan University of Science and Technology N.09001263. 
In this paper, we investigate the numerical method for the Fokker-Planck equation in an infinite channel, which was first introduced by Fokker and Planck to describe the Brownian motion of particles, and has been used in a number of different fields, such as solid-state physics, quantum optics, chemical physics, theoretical biology and circuit theory; e.g., see [3, 27]. The theoretical analysis of this equation can be found in [7, 26.

Let $v$ be the velocity of the particles, $R=\{v \mid-\infty<v<\infty\}, I=\{x|| x \mid<1\}$ and $\Omega=I \times R$ with the boundary $\Gamma=\{(x, v)|| x \mid=1\}$. Moreover, $\Gamma_{1}=$ $\{(x, v) \mid x=-1, v \geq 0$ or $x=1, v \leq 0\}$ and $\Gamma_{2}=\Gamma-\Gamma_{1}$. For fixedness, we focus on Kramers' model with the distribution function in position and velocity space, describing the Brownian motion of particles in an external field with quadratic potential. We also assume that the wall is absorbing. More precisely, the probability of current at $x=-1$ vanishes for the particles leaving the wall into the domain, i.e., for the particles with positive velocity. Similarly, we have the absorbing wall at $x=1$, for the particles with negative velocity. We denote by $W(x, v, t)$ the probability density. The positive constants $k, T, m$ are the Boltzmann's constant, the absolute temperature, and the mass of particles, respectively. Let $\mu=\frac{k T}{m}$ and $\beta_{0}^{-1}>0$ be the particle relaxation time. $\gamma>0$ is the constant in the potential of the external field. For simplicity, we denote $\frac{\partial W}{\partial v}$ by $\partial_{v} W$, etc. Then the considered problem is of the form

$$
\begin{cases}\partial_{t} W(x, v, t)+v \partial_{x} W(x, v, t)-\beta_{0} \partial_{v}(v W(x, v, t)) & \\ +\gamma x \partial_{v} W(x, v, t)-\beta_{0} \mu \partial_{v}^{2} W(x, v, t)=0, & (x, v) \in \Omega, 0<t \leq T, \\ W(x, v, t)=0, & (x, v) \text { on } \Gamma_{1}, 0<t \leq T \\ W(x, v, t) \rightarrow 0, & |v| \rightarrow \infty, 0<t \leq T \\ W(x, v, 0)=W_{0}(x, v), & (x, v) \in \bar{\Omega} .\end{cases}
$$

Some numerical algorithms were proposed for solving (1.1). For instance, Cartling [4] provided finite difference schemes, and Moore and Flaherty [25] considered the Galerkin method. In actual computations, we often imposed an artificial boundary and certain artificial boundary condition, which induces additional numerical errors. So it seems reasonable to solve the Fokker-Planck equation directly.

An interesting and challenging problem is how to use the spectral method for various important partial differential equations of non-standard types on unbounded domains, such as (1.1). Tang, Mckee and Reeks 29] used the Hermite spectral method for a simplified one-dimensional model which is a standard parabolic equation. Recently, Fok, Guo and Tang 8 proposed the Hermite spectral-finite difference scheme for (1.1). However, the finite difference approximation in the $x$ direction seriously limits the numerical accuracy.

The difficulties of dealing with (1.1) numerically are caused by several facts. Firstly, this problem behaves like a parabolic equation in the $v$-direction, and behaves like a hyperbolic equation in the $x$-direction. Thereby, we could not design the spectral schemes and analyze the numerical errors in the usual way. Next, this equation looks like different kinds of hyperbolic equations in the $x$-directions, for $v>0$ and $v \leq 0$, respectively. Consequently, the solution satisfies different kinds of boundary conditions on different subdomains. Therefore, we have to use domain decomposition and different approximations on different unbounded subdomains. Finally, the terms $\partial_{v}^{2} W(x, v, t)$ and $\partial_{x} W(x, v, t)$ in $(1.1)$ possess the coefficients 1 
and $v$ varying from $-\infty$ to $\infty$. This matter also brings some difficulties in actual computation and numerical analysis.

To remedy the deficiencies as discussed before, we develop in this paper the composite generalized Laguerre-Legendre spectral method. We establish some results on the composite approximations, which are the mixed approximations by using generalized Laguerre functions and Legendre polynomials, coupled with domain decomposition. By suitably adjusting the parameters involved in the Laguerre functions, we design reasonable spectral schemes with exactly the same weight functions as in the underlying problems, and fit the asymptotic behaviors of exact solutions properly. In particular, the results on certain quasi-orthogonal approximations play an important role in spectral methods for PDEs on unbounded domains, which behave like parabolic equations in some directions and behave like hyperbolic equations in other directions. They are also appropriate for various spectral methods with domain decomposition. As an important application, we propose the composite spectral scheme for (1.1), with the convergence analysis. An efficient algorithm is provided. The numerical results demonstrate its spectral accuracy in space and coincide well with theoretical analysis. The approximation results and techniques developed in this paper are applicable to many other problems of non-standard types on unbounded domains, as well as exterior problems.

This paper is organized as follows. In the next section, we build up some precise results on the composite generalized Laguerre-Legendre approximations. In section 3 , we design the composite spectral method for (1.1) and prove its convergence. We also describe the implementation of the proposed method and present some numerical results. The final section is for concluding remarks.

\section{Composite generalized Laguerre-Legendre Approximations}

In this section, we establish the basic results on the composite generalized Laguerre-Legendre approximations.

2.1. Generalized Laguerre approximations. We first recall the generalized Laguerre approximation. Let $\Lambda_{1}=\{v \mid 0<v<\infty\}$ and $\chi(v)$ be a certain weight function. For any integer $r \geq 0$,

$$
H_{\chi}^{r}\left(\Lambda_{1}\right)=\left\{u \mid u \text { is measurable on } \Lambda_{1} \text { and }\|u\|_{r, \chi, \Lambda_{1}}<\infty\right\}
$$

equipped with the following inner product, semi-norm and norm:

$$
\begin{gathered}
(u, w)_{r, \chi, \Lambda_{1}}=\sum_{0 \leq k \leq r} \int_{\Lambda_{1}} \partial_{v}^{k} u(v) \partial_{v}^{k} w(v) \chi(v) d v \\
|u|_{r, \chi, \Lambda_{1}}=\int_{\Lambda_{1}}\left(\partial_{v}^{r} u(v)\right)^{2} \chi(v) d v, \quad\|u\|_{r, \chi, \Lambda_{1}}=(u, u)_{r, \chi, \Lambda_{1}}^{\frac{1}{2}} .
\end{gathered}
$$

In particular, $H_{\chi}^{0}\left(\Lambda_{1}\right)=L_{\chi}^{2}\left(\Lambda_{1}\right)$, with the inner product $(u, w)_{\chi}, \Lambda_{1}$ and the norm $\|u\|_{\chi, \Lambda_{1}}$. We omit the subscript $\chi$ in the notation when $\chi(v) \equiv 1$.

Let $\omega_{\alpha, \beta}^{1}(v)=v^{\alpha} e^{-\beta v}, \alpha>-1, \beta>0$ be the generalized Laguerre weight function. The generalized Laguerre polynomial of degree $l$ is defined by

$$
\mathcal{L}_{l}^{(\alpha, \beta)}(v)=\frac{1}{l !} v^{-\alpha} e^{\beta v} \partial_{v}^{l}\left(v^{l+\alpha} e^{-\beta v}\right) .
$$

The set of $\mathcal{L}_{l}^{(\alpha, \beta)}(v)$ is a complete $L_{\omega_{\alpha, \beta}^{1}}^{2}\left(\Lambda_{1}\right)$-orthogonal system. 
For any integer $N \geq 0, \mathcal{P}_{N}\left(\Lambda_{1}\right)$ stands for the set of all polynomials of degree at most $N$. The orthogonal projection $P_{N, \alpha, \beta, \Lambda_{1}}: L_{\omega_{\alpha, \beta}^{1}}^{2}\left(\Lambda_{1}\right) \longrightarrow \mathcal{P}_{N}\left(\Lambda_{1}\right)$ is defined by

$$
\left(P_{N, \alpha, \beta, \Lambda_{1}} u-u, \phi\right)_{\omega_{\alpha, \beta}^{1}, \Lambda_{1}}=0, \quad \forall \phi \in \mathcal{P}_{N}\left(\Lambda_{1}\right)
$$

If $\partial_{v}^{r} u \in L_{\omega_{\alpha+r, \beta}^{1}}^{2}\left(\Lambda_{1}\right)$ and integers $0 \leq s \leq r$, then (cf. [22])

$$
\left\|\partial_{v}^{s}\left(P_{N, \alpha, \beta, \Lambda_{1}} u-u\right)\right\|_{\omega_{\alpha+s, \beta}^{1}, \Lambda_{1}} \leq c(\beta N)^{\frac{s-r}{2}}\left\|\partial_{v}^{r} u\right\|_{\omega_{\alpha+r, \beta}^{1}, \Lambda_{1}}
$$

Hereafter, $c$ denotes a generic positive constant independent of $M, N, \beta$ and any function.

To design a proper spectral method for the Fokker-Planck equation and many other problems, we shall use the orthogonal system of generalized Laguerre functions, defined by

$$
\tilde{\mathcal{L}}_{l}^{(\alpha, \beta)}(v)=e^{-\frac{1}{2} \beta v} \mathcal{L}_{l}^{(\alpha, \beta)}(v), \quad l=0,1,2, \ldots
$$

The set of $\tilde{\mathcal{L}}_{l}^{(\alpha, \beta)}(v)$ is a complete $L_{v^{\alpha}}^{2}\left(\Lambda_{1}\right)$-orthogonal system.

Next, let $\mathcal{Q}_{N, \beta}\left(\Lambda_{1}\right)=\left\{e^{-\frac{1}{2} \beta v} \psi \mid \psi \in \mathcal{P}_{N}\left(\Lambda_{1}\right)\right\}$. The orthogonal projection $\tilde{P}_{N, \alpha, \beta, \Lambda_{1}}: L_{v^{\alpha}}^{2}\left(\Lambda_{1}\right) \rightarrow \mathcal{Q}_{N, \beta}\left(\Lambda_{1}\right)$ is defined by

$$
\left(\tilde{P}_{N, \alpha, \beta, \Lambda_{1}} u-u, \phi\right)_{v^{\alpha}, \Lambda_{1}}=0, \quad \forall \phi \in \mathcal{Q}_{N, \beta}\left(\Lambda_{1}\right) .
$$

Since $\tilde{P}_{N, \alpha, \beta, \Lambda_{1}} u=e^{-\frac{1}{2} \beta v} P_{N, \alpha, \beta, \Lambda_{1}}\left(e^{\frac{1}{2} \beta v} u\right)$, we know from $(2.2)$ that if $u \in L_{v^{\alpha}}^{2}\left(\Lambda_{1}\right)$, $\partial_{v}^{r}\left(e^{\frac{1}{2} \beta v} u\right) \in L_{\omega_{r+\alpha, \beta}^{1}}^{2}\left(\Lambda_{1}\right)$ and $r \geq 0$ is an integer, then

$$
\left\|\tilde{P}_{N, \alpha, \beta, \Lambda_{1}} u-u\right\|_{v^{\alpha}, \Lambda_{1}} \leq c(\beta N)^{-\frac{r}{2}}\left\|\partial_{v}^{r}\left(e^{\frac{1}{2} \beta v} u\right)\right\|_{\omega_{\alpha+r, \beta}^{1}, \Lambda_{1}} .
$$

Remark 2.1. If $u \in H_{v^{\alpha+r}}^{r}\left(\Lambda_{1}\right)$, then the norm $\left\|\partial_{v}^{r}\left(e^{\frac{1}{2} \beta v} u\right)\right\|_{\omega_{r+\alpha, \beta}^{1}, \Lambda_{1}}$ is finite.

For parabolic equations and differential equations which behave like parabolic equations in the $v$-direction, we may approximate the initial state by using the above projection. But for spectral methods coupled with domain decompositions, we have to match the functions on the common boundaries of adjacent subdomains. To do this, we introduce the spaces

$$
\begin{gathered}
\mathcal{F}\left(\Lambda_{1}\right)=L^{2}\left(\Lambda_{1}\right) \cap\{u \mid \text { there exists a finite trace of } u \text { at } v=0\}, \\
{ }_{0} \mathcal{F}\left(\Lambda_{1}\right)=\left\{u \mid u \in \mathcal{F}\left(\Lambda_{1}\right) \text { and } u(0)=0\right\}, \\
{ }_{0} \mathcal{Q}_{N, \beta}\left(\Lambda_{1}\right)=\left\{\phi \mid \phi \in \mathcal{Q}_{N, \beta}\left(\Lambda_{1}\right) \text { and } \phi(0)=0\right\} .
\end{gathered}
$$

The set $\mathcal{F}\left(\Lambda_{1}\right)$ is meaningful. For instance, if $u \in L^{2}\left(\Lambda_{1}\right)$ and $u$ is continuous near the point $v=0$, then $u \in \mathcal{F}\left(\Lambda_{1}\right)$. The orthogonal projection ${ }_{0} \tilde{P}_{N, \beta, \Lambda_{1}}:{ }_{0} \mathcal{F}\left(\Lambda_{1}\right) \rightarrow$ ${ }_{0} \mathcal{Q}_{N, \beta}\left(\Lambda_{1}\right)$ is defined by

$$
\left({ }_{0} \tilde{P}_{N, \beta, \Lambda_{1}} u-u, \phi\right)_{\Lambda_{1}}=0, \quad \forall \phi \in{ }_{0} \mathcal{Q}_{N, \beta}\left(\Lambda_{1}\right) .
$$

For any $u \in \mathcal{F}\left(\Lambda_{1}\right)$, we set $\tilde{u}(v)=u(v)-u(0) e^{-\frac{1}{2} \beta v}$ and introduce the quasiorthogonal projection

$$
{ }_{*} \tilde{P}_{N, \beta, \Lambda_{1}} u(v)={ }_{0} \tilde{P}_{N, \beta, \Lambda_{1}} \tilde{u}(v)+u(0) e^{-\frac{1}{2} \beta v} .
$$

We now estimate $\left\|{ }_{*} \tilde{P}_{N, \beta, \Lambda_{1}} u-u\right\|_{\Lambda_{1}}$. Firstly, by the projection theorem,

$$
\left\|\tilde{P}_{N, \beta, \Lambda_{1}} \tilde{u}-\tilde{u}\right\|_{\Lambda_{1}} \leq\|\phi-\tilde{u}\|_{\Lambda_{1}}, \quad \forall \phi \in{ }_{0} \mathcal{Q}_{N, \beta}\left(\Lambda_{1}\right) .
$$


We take

$$
\phi(v)=e^{-\frac{1}{2} \beta v} \int_{0}^{v} P_{N-1,0, \beta, \Lambda_{1}} \partial_{\xi}\left(e^{\frac{1}{2} \beta \xi} \tilde{u}(\xi)\right) d \xi \in{ }_{0} \mathcal{Q}_{N, \beta}\left(\Lambda_{1}\right) .
$$

Like the derivation of (3.3) of [15], we can use integration by parts to show that for any $w \in{ }_{0} \mathcal{F}\left(\Lambda_{1}\right) \cap H^{1}\left(\Lambda_{1}\right)$,

$$
\left\|\partial_{v} w\right\|_{\Lambda_{1}}^{2}+\frac{1}{4} \beta^{2}\|w\|_{\Lambda_{1}}^{2}=\left\|\partial_{v}\left(e^{\frac{1}{2} \beta v} w\right)\right\|_{\omega_{0, \beta}^{1}, \Lambda_{1}}^{2} .
$$

Let $w(v)=\phi(v)-\tilde{u}(v)$. Then, along with $(2.2)$, a direct calculation yields that

$$
\begin{aligned}
\|\phi-\tilde{u}\|_{\Lambda_{1}} & \leq c \beta^{-1}\left\|P_{N-1,0, \beta, \Lambda_{1}} \partial_{v}\left(e^{\frac{1}{2} \beta v} \tilde{u}\right)-\partial_{v}\left(e^{\frac{1}{2} \beta v} \tilde{u}\right)\right\|_{\omega_{0, \beta}^{1}, \Lambda_{1}} \\
& \leq c \beta^{-1}(\beta N)^{-\frac{r}{2}}\left\|\partial_{v}^{r+1}\left(e^{\frac{1}{2} \beta v} \tilde{u}\right)\right\|_{\omega_{r, \beta}^{1}, \Lambda_{1}},
\end{aligned}
$$

whence

$$
\left\|\tilde{P}_{N, \beta, \Lambda_{1}} \tilde{u}-\tilde{u}\right\|_{\Lambda_{1}} \leq c \beta^{-1}(\beta N)^{-\frac{r}{2}}\left\|\partial_{v}^{r+1}\left(e^{\frac{1}{2} \beta v} \tilde{u}\right)\right\|_{\omega_{r, \beta}^{1}, \Lambda_{1}} .
$$

It can be checked that ${ }_{*} \tilde{P}_{N, \beta, \Lambda_{1}} u-u={ }_{0} \tilde{P}_{N, \beta, \Lambda_{1}} \tilde{u}-\tilde{u}$. Therefore, we derive that for integer $r \geq 0$,

$$
\begin{aligned}
& \left\|\tilde{P}_{*, \beta, \Lambda_{1}} u-u\right\|_{\Lambda_{1}}=\|\|_{0} \tilde{P}_{N, \beta, \Lambda_{1}} \tilde{u}-\tilde{u} \|_{\Lambda_{1}} \\
& \quad \leq c \beta^{-1}(\beta N)^{-\frac{r}{2}}\left\|\partial_{v}^{r+1}\left(e^{\frac{1}{2} \beta v} \tilde{u}\right)\right\|_{\omega_{r, \beta}^{1}, \Lambda_{1}} \\
& \quad=c \beta^{-1}(\beta N)^{-\frac{r}{2}}\left\|\partial_{v}^{r+1}\left(e^{\frac{1}{2} \beta v} u\right)\right\|_{\omega_{r, \beta}^{1}, \Lambda_{1}} .
\end{aligned}
$$

Remark 2.2. For dealing with the boundary condition at $v=0$, we used the projection ${ }_{0} \tilde{P}_{N, \beta, \Lambda_{1}}$ to define and analyze the projection ${ }_{*} \tilde{P}_{N, \beta, \Lambda_{1}}$, instead of the usual projection ${ }_{0} P_{N, \beta, \Lambda_{1}}$. In fact, ${ }_{0} P_{N, \beta, \Lambda_{1}}$ is an orthogonal projection defined on $\Lambda_{1}$, with the weight function $e^{-\beta v}$. However, the underlying problem (1.1) is not wellposed in the Sobolev space with such a weight function. Thus, we used the projection ${ }_{0} \tilde{P}_{N, \alpha, \beta, \Lambda_{1}}$ to define ${ }_{*} \tilde{P}_{N, \beta, \Lambda_{1}}$ on $\Lambda_{1}$, and we introduce the projection ${ }_{*} \tilde{P}_{N, \alpha, \beta, \Lambda_{2}}$ on $\Lambda_{2}$ similarly, in the next part. They are orthogonal projections with the same weight function as in (1.1), and so are the most appropriate for its numerical simulation. On the other hand, we may approximate the initial value $W_{0} \in L_{v^{2}+1}^{2}(\Omega)$ of (1.1) by a combination of such projections. But for smooth $W_{0}$, we can also use a combination of orthogonal projections in a certain weighted $H^{1}(\Omega)$-norm.

In the numerical analysis of spectral methods, we need some orthogonal projections in $H^{1}$-norms, which correspond to the considered differential equations. If the powers of $v$ appearing in the coefficients of derivatives of higher orders are not smaller than those of derivatives of lower orders, then it is easier to estimate the errors of the related approximations. But in the Fokker-Planck equation, there exist the terms $-\beta_{0} \mu \partial_{v}^{2} W(x, v, t),-\beta_{0} v \partial_{v} W(x, v, t)$ and so on. Thereby, we are forced to study certain unusual approximations. To do this, we set

$$
\begin{gathered}
H_{\chi_{1}, \chi_{0}}^{1}\left(\Lambda_{1}\right)=\left\{u \mid u \in L_{\chi_{0}}^{2}\left(\Lambda_{1}\right) \text { and } \partial_{v} u \in L_{\chi_{1}}^{2}\left(\Lambda_{1}\right)\right\}, \\
{ }_{0} H_{\chi_{1}, \chi_{0}}^{1}\left(\Lambda_{1}\right)=\left\{u \mid u \in H_{\chi_{1}, \chi_{0}}^{1}\left(\Lambda_{1}\right) \text { and } u(0)=0\right\} .
\end{gathered}
$$

Let ${ }_{0} \mathcal{P}_{N}\left(\Lambda_{1}\right)=\left\{u \mid u \in \mathcal{P}_{N}\left(\Lambda_{1}\right)\right.$ and $\left.u(0)=0\right\}$ and $\xi_{\beta}^{1}(v)=\left(v^{2}+1\right) e^{-\beta v}$. We introduce the auxiliary orthogonal projection ${ }_{0} \bar{P}_{N, \beta, \Lambda_{1}}^{1}:{ }_{0} H_{\omega_{0, \beta}^{1}, \xi_{\beta}^{1}}^{1}\left(\Lambda_{1}\right) \rightarrow{ }_{0} \mathcal{P}_{N}\left(\Lambda_{1}\right)$, defined by

$$
\left(\partial_{v}\left({ }_{0} \bar{P}_{N, \beta, \Lambda_{1}}^{1} u-u\right), \partial_{v} \phi\right)_{\omega_{0, \beta}^{1}, \Lambda_{1}}+\left({ }_{0} \bar{P}_{N, \beta, \Lambda_{1}}^{1} u-u, \phi\right)_{\xi_{\beta}^{1}, \Lambda_{1}}=0, \quad \forall \phi \in{ }_{0} \mathcal{P}_{N}\left(\Lambda_{1}\right) .
$$


It is noted that in the above definition, the power of $v$ involved in the weight function for the function is even greater than that for its derivative!

For any $u \in H_{1, v^{2}+1}^{1}\left(\Lambda_{1}\right)$, we set $\tilde{u}(v)=u(v)-u(0) e^{-\frac{1}{2} \beta v}$. Obviously, $\tilde{u} \in$ ${ }_{0} H_{1, v^{2}+1}^{1}\left(\Lambda_{1}\right)$. The quasi-orthogonal projection, which plays an important role in the forthcoming analysis, is defined by

$$
P_{N, \beta, \Lambda_{1}}^{1} u=e^{-\frac{1}{2} \beta v}\left({ }_{0} \bar{P}_{N, \beta, \Lambda_{1}}^{1}\left(e^{\frac{1}{2} \beta v} \tilde{u}\right)+u(0)\right) \in \mathcal{Q}_{N, \beta}\left(\Lambda_{1}\right) .
$$

In order to derive approximation results, we recall the following fact (cf. Lemma 2.2 of [22]).

Proposition 2.1. If $w \in H_{\omega_{\alpha, \beta}^{1}}^{1}\left(\Lambda_{1}\right), w(0)=0$ and $\alpha<1$, then

$$
\|w\|_{\omega_{\alpha, \beta}^{1}, \Lambda_{1}}^{2} \leq c_{\alpha, \beta}\left\|\partial_{v} w\right\|_{\omega_{\alpha, \beta}^{1}, \Lambda_{1}}^{2},
$$

where $c_{\alpha, \beta}=\frac{4}{\beta^{2}}$ for $\alpha \leq 0$ and $c_{\alpha, \beta}=\frac{4-2 \alpha}{\beta^{2}(1-\alpha)}$ for $0<\alpha<1$. Moreover, for $w \in H_{\omega_{\alpha, \beta}^{1}}^{1}\left(\Lambda_{1}\right) \cap L_{\omega_{\alpha-2, \beta}^{1}}^{2}\left(\Lambda_{1}\right)$ and $\alpha>1$,

$$
\|w\|_{\omega_{\alpha, \beta}^{1}, \Lambda_{1}}^{2} \leq \frac{2(3 \alpha-2)}{\beta^{2}(\alpha-1)}\left\|\partial_{v} w\right\|_{\omega_{\alpha, \beta}^{1}, \Lambda_{1}}^{2}+\frac{4 \alpha(\alpha-1)}{\beta^{2}}\|w\|_{\omega_{\alpha-2, \beta}^{1}, \Lambda_{1}}^{2} .
$$

Lemma 2.1. If $u \in H_{1, v^{2}+1}^{1}\left(\Lambda_{1}\right), \partial_{v}^{r+1}\left(e^{\frac{1}{2} \beta v} u\right) \in L_{\omega_{r+1, \beta}^{1}}^{2}\left(\Lambda_{1}\right)$ and $r \geq 1$ is an integer, then

$$
\begin{aligned}
& \left\|\partial_{v}\left(P_{N, \beta, \Lambda_{1}}^{1} u-u\right)\right\|_{\Lambda_{1}}+\left\|P_{N, \beta, \Lambda_{1}}^{1} u-u\right\|_{v^{2}+1, \Lambda_{1}} \\
& \leq c\left(\beta+\beta^{-2}\right)(\beta N)^{\frac{1-r}{2}}\left\|\partial_{v}^{r+1}\left(e^{\frac{1}{2} \beta v} u\right)\right\|_{\omega_{r+1, \beta}^{1}, \Lambda_{1}} .
\end{aligned}
$$

Proof. The proof is divided into four steps.

We first consider the auxiliary orthogonal projection $P_{N, \beta, \Lambda_{1}}^{1, *}: H_{\omega_{2, \beta}^{1}, \omega_{0, \beta}^{1}}^{1}\left(\Lambda_{1}\right) \rightarrow$ $\mathcal{P}_{N}\left(\Lambda_{1}\right)$, defined by

$$
\left(\partial_{v}\left(P_{N, \beta, \Lambda_{1}}^{1, *} w-w\right), \partial_{v} \phi\right)_{\omega_{2, \beta}^{1}, \Lambda_{1}}+\left(P_{N, \beta, \Lambda_{1}}^{1, *} w-w, \phi\right)_{\omega_{0, \beta}^{1}, \Lambda_{1}}=0, \quad \forall \phi \in \mathcal{P}_{N}\left(\Lambda_{1}\right) .
$$

According to Theorem 2.2 of [22],

$$
\begin{aligned}
& \left\|\partial_{v}\left(P_{N, \beta, \Lambda_{1}}^{1, *} w-w\right)\right\|_{\omega_{2, \beta}^{1}, \Lambda_{1}}+\left\|P_{N, \beta, \Lambda_{1}}^{1, *} w-w\right\|_{\omega_{0, \beta}^{1}, \Lambda_{1}} \\
& \quad \leq c(\beta N)^{\frac{1-r}{2}}\left\|\partial_{v}^{r} w\right\|_{\omega_{r+1, \beta}^{1}, \Lambda_{1}} .
\end{aligned}
$$

Next, by the projection theorem, for any $w \in{ }_{0} H_{\omega_{0, \beta}^{1}, \xi_{\beta}^{1}}^{1}\left(\Lambda_{1}\right)$,

$$
\begin{aligned}
& \left\|\partial_{v}\left({ }_{0} \bar{P}_{N, \beta, \Lambda_{1}}^{1} w-w\right)\right\|_{\omega_{0, \beta}^{1}, \Lambda_{1}}+\|\|_{0} \bar{P}_{N, \beta, \Lambda_{1}}^{1} w-w \|_{\xi_{\beta}^{1}, \Lambda_{1}}^{2} \\
& \leq\left\|\partial_{v}(\phi-w)\right\|_{\omega_{0, \beta}^{1}, \Lambda_{1}}+\|\phi-w\|_{\xi_{\beta}^{1}, \Lambda_{1}}^{2}, \forall \phi \in{ }_{0} \mathcal{P}_{N}\left(\Lambda_{1}\right) .
\end{aligned}
$$

We now estimate the upper-bound of the right side of the above inequality. We take

$$
\phi(v)=\int_{0}^{v} P_{N-1, \beta, \Lambda_{1}}^{1, *}\left(\partial_{\xi} w(\xi)\right) d \xi \in{ }_{0} \mathcal{P}_{N}\left(\Lambda_{1}\right) .
$$

By (2.6),

$$
\begin{aligned}
& \left\|\partial_{v}(\phi-w)\right\|_{\omega_{0, \beta}^{1}, \Lambda_{1}}=\left\|P_{N-1, \beta, \Lambda_{1}}^{1, *}\left(\partial_{v} w\right)-\partial_{v} w\right\|_{\omega_{0, \beta}^{1}, \Lambda_{1}} \\
& \quad \leq c(\beta N)^{\frac{1-r}{2}}\left\|\partial_{v}^{r+1} w\right\|_{\omega_{r+1, \beta}^{1}, \Lambda_{1}} .
\end{aligned}
$$


Moreover, by using (2.6), (2.7) and the second result of Proposition 2.1, we deduce that $(2.8)$

$$
\begin{aligned}
\left\|\partial_{v}(\phi-w)\right\|_{\omega_{2, \beta}^{1}, \Lambda_{1}} & \leq \frac{c}{\beta}\left(\left\|\partial_{v}^{2}(\phi-w)\right\|_{\omega_{2, \beta}^{1}, \Lambda_{1}}+\left\|\partial_{v}(\phi-w)\right\|_{\omega_{0, \beta}^{1}, \Lambda_{1}}\right) \\
& =\frac{c}{\beta}\left(\left\|\partial_{v}\left(P_{N-1, \beta, \Lambda_{1}}^{1, *}\left(\partial_{v} w\right)-\partial_{v} w\right)\right\|_{\omega_{2, \beta}^{1}, \Lambda_{1}}+\left\|\partial_{v}(\phi-w)\right\|_{\omega_{0, \beta}^{1}, \Lambda_{1}}\right) \\
& \leq \frac{c}{\beta}(\beta N)^{\frac{1-r}{2}}\left\|\partial_{v}^{r+1} w\right\|_{\omega_{r+1, \beta}^{1}, \Lambda_{1}} .
\end{aligned}
$$

On the other hand, by virtue of (2.7) and the first result of Proposition 2.1, we obtain that

$$
\|\phi-w\|_{\omega_{0, \beta}^{1}, \Lambda_{1}} \leq \frac{2}{\beta}\left\|\partial_{v}(\phi-w)\right\|_{\omega_{0, \beta}^{1}, \Lambda_{1}} \leq \frac{c}{\beta}(\beta N)^{\frac{1-r}{2}}\left\|\partial_{v}^{r+1} w\right\|_{\omega_{r+1, \beta}^{1}, \Lambda_{1}} .
$$

Furthermore, thanks to (2.8), (2.9) and the second result of Proposition 2.1, we verify that

$$
\begin{aligned}
\|\phi-w\|_{\omega_{2, \beta}^{1}, \Lambda_{1}} & \leq \frac{c}{\beta}\left(\left\|\partial_{v}(\phi-w)\right\|_{\omega_{2, \beta}^{1}, \Lambda_{1}}+\|\phi-w\|_{\omega_{0, \beta}^{1}, \Lambda_{1}}\right) \\
& \leq \frac{c}{\beta^{2}}(\beta N)^{\frac{1-r}{2}}\left\|\partial_{v}^{r+1} w\right\|_{\omega_{r+1, \beta}^{1}, \Lambda_{1}} .
\end{aligned}
$$

A combination of the above estimate with (2.7) and (2.9) gives that

$$
\begin{aligned}
\left\|\partial_{v}\left({ }_{0} \bar{P}_{N, \beta, \Lambda_{1}}^{1} w-w\right)\right\|_{\omega_{0, \beta}^{1}, \Lambda_{1}} & +\|\|_{0} \bar{P}_{N, \beta, \Lambda_{1}}^{1} w-w \|_{\xi_{\beta}^{1}, \Lambda_{1}} \\
& \leq c\left(1+\frac{1}{\beta^{2}}\right)(\beta N)^{\frac{1-r}{2}}\left\|\partial_{v}^{r+1} w\right\|_{\omega_{r+1, \beta}^{1}, \Lambda_{1}} .
\end{aligned}
$$

Now, we are in a position of deriving the desired result. A direct calculation shows that

$$
P_{N, \beta, \Lambda_{1}}^{1} u-u=e^{-\frac{1}{2} \beta v}\left({ }_{0} \bar{P}_{N, \beta, \Lambda_{1}}^{1}\left(e^{\frac{1}{2} \beta v} \tilde{u}\right)-e^{\frac{1}{2} \beta v} \tilde{u}\right) .
$$

This fact along with (2.10) leads to

$$
\begin{aligned}
\left\|P_{N, \beta, \Lambda_{1}}^{1} u-u\right\|_{v^{2}+1, \Lambda_{1}} & =\left\|\bar{P}_{N, \beta, \Lambda_{1}}^{1}\left(e^{\frac{1}{2} \beta v} \tilde{u}\right)-e^{\frac{1}{2} \beta v} \tilde{u}\right\|_{\xi_{\beta}^{1}, \Lambda_{1}} \\
& \leq c\left(1+\beta^{-2}\right)(\beta N)^{\frac{1-r}{2}}\left\|\partial_{v}^{r+1}\left(e^{\frac{1}{2} \beta v} \tilde{u}\right)\right\|_{\omega_{r+1, \beta}^{1}, \Lambda_{1}} \\
& =c\left(1+\beta^{-2}\right)(\beta N)^{\frac{1-r}{2}}\left\|\partial_{v}^{r+1}\left(e^{\frac{1}{2} \beta v} u\right)\right\|_{\omega_{r+1, \beta}^{1}, \Lambda_{1}} .
\end{aligned}
$$

Clearly,

$$
\begin{aligned}
& \partial_{v}\left(P_{N, \beta, \Lambda_{1}}^{1} u-u\right) \\
& =e^{-\frac{1}{2} \beta v} \partial_{v}\left({ }_{0} \bar{P}_{N, \beta, \Lambda_{1}}^{1}\left(e^{\frac{1}{2} \beta v} \tilde{u}\right)-e^{\frac{1}{2} \beta v} \tilde{u}\right)-\frac{1}{2} \beta e^{-\frac{1}{2} \beta v}\left({ }_{0} \bar{P}_{N, \beta, \Lambda_{1}}^{1}\left(e^{\frac{1}{2} \beta v} \tilde{u}\right)-e^{\frac{1}{2} \beta v} \tilde{u}\right) .
\end{aligned}
$$

Accordingly, using (2.10) again gives that

$$
\begin{aligned}
\left\|\partial_{v}\left(P_{N, \beta, \Lambda_{1}}^{1} u-u\right)\right\|_{\Lambda_{1} \leq} & \left\|\partial_{v}\left({ }_{0} \bar{P}_{N, \beta, \Lambda_{1}}^{1}\left(e^{\frac{1}{2} \beta v} \tilde{u}\right)-e^{\frac{1}{2} \beta v} \tilde{u}\right)\right\|_{\omega_{0, \beta}^{1}, \Lambda_{1}} \\
& +\beta\|\|_{0} \bar{P}_{N, \beta, \Lambda_{1}}^{1}\left(e^{\frac{1}{2} \beta v} \tilde{u}\right)-e^{\frac{1}{2} \beta v} \tilde{u} \|_{\omega_{0, \beta}^{1}, \Lambda_{1}} \\
\leq & c\left(\beta+\beta^{-2}\right)(\beta N)^{\frac{1-r}{2}}\left\|\partial_{v}^{r+1}\left(e^{\frac{1}{2} \beta v} u\right)\right\|_{\omega_{r+1, \beta}^{1}, \Lambda_{1}} .
\end{aligned}
$$

This ends the proof.

Remark 2.3. In many problems, the powers of $v$ appearing in the coefficients of derivatives of higher orders are not smaller than those of derivatives of lower orders. For instance, we consider the following Black-Sholes-like equation:

$$
\partial_{t} W-\partial_{v}\left(v^{2} \partial_{v} W\right)+W=f, \quad 0<v<\infty,
$$


with the homogeneous boundary condition at $v=0, \infty$. Let $\mathcal{M}=\left\{u \mid u, v \partial_{v} u \in\right.$ $\left.L^{2}(0, \infty)\right\}$. Then the weak formulation of the above problem is to find $W \in \mathcal{M}$ such that

$$
\int_{0}^{\infty} \partial_{t} W u d v+\int_{0}^{\infty} v^{2} \partial_{v} W \partial_{v} u d v+\int_{0}^{\infty} W u d v=\int_{0}^{\infty} f u d v, \quad \forall u \in \mathcal{M} .
$$

Let $\mathcal{M}_{N}$ be a certain $N$-dimensional subspace of $\mathcal{M}$. The corresponding spectral method is to seek $w_{N} \in \mathcal{M}_{N}$ such that

$$
\int_{0}^{\infty} \partial_{t} w_{N} \phi d v+\int_{0}^{\infty} v^{2} \partial_{v} w_{N} \partial_{v} \phi d v+\int_{0}^{\infty} w_{N} \phi d v=\int_{0}^{\infty} f \phi d v, \quad \forall \phi \in \mathcal{M}_{N} .
$$

In numerical analysis, we need the orthogonal projection $P_{N}^{*}: \mathcal{M} \rightarrow \mathcal{M}_{N}$ such that

$$
\int_{0}^{\infty} v^{2} \partial_{v}\left(P_{N}^{*} u-u\right) \partial_{v} \phi d v+\int_{0}^{\infty}\left(P_{N}^{*} u-u\right) \phi d v=0, \quad \forall \phi \in \mathcal{M}_{N}
$$

For deriving the approximation error, we can use a Poincaré-type inequality, i.e., the Hardy-type inequality:

$$
\int_{0}^{\infty} u^{2} d v \leq c \int_{0}^{\infty} v^{2}\left(\partial_{v} u\right)^{2} d v
$$

But it does not work in some PDEs, such as the Fokker-Planck equation. Thereby, we are forced to consider the unusual approximations as in the last paragraph.

We now consider some approximations on the interval $\Lambda_{2}=(-\infty, 0)$. The space $H_{\chi}^{r}\left(\Lambda_{2}\right)$ is defined as usual, with the inner product $(u, w)_{r, \chi, \Lambda_{2}}$, the semi-norm $|u|_{r, \chi, \Lambda_{2}}$ and the norm $\|u\|_{r, \chi, \Lambda_{2}}$, especially, $H_{\chi}^{0}\left(\Lambda_{2}\right)=L_{\chi}^{2}\left(\Lambda_{2}\right)$, with the inner product $(u, w)_{\chi, \Lambda_{2}}$ and the norm $\|u\|_{\chi, \Lambda_{2}}$.

Let $\mathcal{Q}_{N, \beta}\left(\Lambda_{2}\right)=\left\{e^{\frac{1}{2} \beta v} \psi \mid \psi \in \mathcal{P}_{N}\left(\Lambda_{2}\right)\right\}$. The orthogonal projection $\tilde{P}_{N, \alpha, \beta, \Lambda_{2}}$ : $L_{(-v)^{\alpha}}^{2}\left(\Lambda_{2}\right) \rightarrow \mathcal{Q}_{N, \beta}\left(\Lambda_{2}\right)$ is defined by

$$
\left(\tilde{P}_{N, \alpha, \beta, \Lambda_{2}} u-u, \phi\right)_{(-v)^{\alpha}, \Lambda_{2}}=0, \quad \forall \phi \in \mathcal{Q}_{N, \beta}\left(\Lambda_{2}\right) .
$$

Let $\omega_{\alpha, \beta}^{2}(v)=\omega_{\alpha, \beta}^{1}(-v)=(-v)^{\alpha} e^{\beta v}$. We can prove as in the derivation of (2.4) that for any integer $r \geq 0$,

$$
\left\|\tilde{P}_{N, \alpha, \beta, \Lambda_{2}} u-u\right\|_{(-v)^{\alpha}, \Lambda_{2}} \leq c(\beta N)^{-\frac{r}{2}}\left\|\partial_{v}^{r}\left(e^{-\frac{1}{2} \beta v} u\right)\right\|_{\omega_{\alpha+r, \beta}^{2}, \Lambda_{2}} .
$$

We set

$$
\begin{gathered}
\mathcal{F}\left(\Lambda_{2}\right)=L^{2}\left(\Lambda_{2}\right) \cap\{u \mid \text { there exists a finite trace of } u \text { at } v=0\}, \\
{ }^{0} \mathcal{F}\left(\Lambda_{2}\right)=\left\{u \mid u \in \mathcal{F}\left(\Lambda_{2}\right) \text { and } u(0)=0\right\}, \\
{ }^{0} \mathcal{Q}_{N, \beta}\left(\Lambda_{2}\right)=\left\{\phi \mid \phi \in \mathcal{Q}_{N, \beta}\left(\Lambda_{2}\right) \text { and } \phi(0)=0\right\} .
\end{gathered}
$$

The orthogonal projection ${ }^{0} \tilde{P}_{N, \beta, \Lambda_{2}}:{ }^{0} \mathcal{F}\left(\Lambda_{2}\right) \rightarrow{ }^{0} \mathcal{Q}_{N, \beta}\left(\Lambda_{2}\right)$ is defined by

$$
\left({ }^{0} \tilde{P}_{N, \beta, \Lambda_{2}} u-u, \phi\right)_{\Lambda_{2}}=0, \quad \forall \phi \in{ }^{0} \mathcal{Q}_{N, \beta}\left(\Lambda_{2}\right) .
$$

For any $u \in \mathcal{F}\left(\Lambda_{2}\right)$, we set $\tilde{u}(v)=u(v)-u(0) e^{\frac{1}{2} \beta v}$, and introduce the following quasi-orthogonal projection:

$$
{ }_{*} \tilde{P}_{N, \beta, \Lambda_{2}} u(v)={ }^{0} \tilde{P}_{N, \beta, \Lambda_{2}} \tilde{u}(v)+u(0) e^{\frac{1}{2} \beta v} .
$$

Like (2.5), if $u \in \mathcal{F}\left(\Lambda_{2}\right), \partial_{v}^{r}\left(e^{-\frac{1}{2} \beta v} u\right) \in L_{\omega_{r, \beta}^{2}}^{2}\left(\Lambda_{2}\right)$ and $r \geq 0$ is an integer, then

$$
\left\|{ }_{*} \tilde{P}_{N, \beta, \Lambda_{2}} u-u\right\|_{\Lambda_{2}} \leq c \beta^{-1}(\beta N)^{-\frac{r}{2}}\left\|\partial_{v}^{r}\left(e^{-\frac{1}{2} \beta v} u\right)\right\|_{\omega_{r, \beta}^{2}, \Lambda_{2}} .
$$


Furthermore, let

$$
\begin{gathered}
H_{\chi_{1}, \chi_{0}}^{1}\left(\Lambda_{2}\right)=\left\{u \mid u \in L_{\chi_{0}}^{2}\left(\Lambda_{2}\right) \text { and } \partial_{v} u \in L_{\chi_{1}}^{2}\left(\Lambda_{2}\right)\right\}, \\
{ }^{0} H_{\chi_{1}, \chi_{0}}^{1}\left(\Lambda_{2}\right)=\left\{u \mid u \in H_{\chi_{1}, \chi_{0}}^{1}\left(\Lambda_{2}\right) \text { and } u(0)=0\right\} .
\end{gathered}
$$

Moreover, ${ }^{0} \mathcal{P}_{N}\left(\Lambda_{2}\right)=\left\{\phi \mid \phi \in \mathcal{P}_{N}\left(\Lambda_{2}\right)\right.$ and $\left.u(0)=0\right\}$ and $\xi_{\beta}^{2}(v)=\left(v^{2}+1\right) e^{\beta v}$. We introduce the auxiliary orthogonal projection ${ }^{0} \bar{P}_{N, \beta, \Lambda_{2}}^{1}:{ }^{0} H_{\omega_{0, \beta}^{2}, \xi_{\beta}^{2}}^{1}\left(\Lambda_{2}\right) \rightarrow{ }^{0} \mathcal{P}_{N}\left(\Lambda_{2}\right)$, defined by

$$
\left(\partial_{v}\left({ }^{0} \bar{P}_{N, \beta, \Lambda_{2}}^{1} u-u\right), \partial_{v} \phi\right)_{\omega_{0, \beta}^{2}, \Lambda_{2}}+\left({ }^{0} \bar{P}_{N, \beta, \Lambda_{2}}^{1} u-u, \phi\right)_{\xi_{\beta}^{2}, \Lambda_{2}}=0, \quad \forall \phi \in{ }^{0} \mathcal{P}_{N}\left(\Lambda_{2}\right) .
$$

For any $u \in H_{1, v^{2}+1}^{1}\left(\Lambda_{2}\right)$, we set $\tilde{u}(v)=u(v)-u(0) e^{\frac{1}{2} \beta v}$. Obviously, we have $\tilde{u} \in$ ${ }^{0} H_{1, v^{2}+1}^{1}\left(\Lambda_{2}\right)$. The quasi-orthogonal projection, which also plays an important role in the analysis, is defined by

$$
P_{N, \beta, \Lambda_{2}}^{1} u=e^{\frac{1}{2} \beta v 0} \bar{P}_{N, \beta, \Lambda_{2}}^{1}\left(e^{-\frac{1}{2} \beta v} u\right) \in \mathcal{Q}_{N, \beta}\left(\Lambda_{2}\right) .
$$

Following the same line as in the proof of Lemma 2.1, we verify that if $u \in$ $H_{1, v^{2}+1}^{1}\left(\Lambda_{2}\right), \partial_{v}^{r+1}\left(e^{-\frac{1}{2} \beta v} u\right) \in L_{\omega_{r+1, \beta}^{2}}^{2}\left(\Lambda_{2}\right)$ and $r \geq 1$ is an integer, then

$$
\begin{aligned}
\left\|\partial_{v}\left(P_{N, \beta, \Lambda_{2}}^{1} u-u\right)\right\|_{\Lambda_{2}} & +\left\|P_{N, \beta, \Lambda_{2}}^{1} u-u\right\|_{v^{2}+1, \Lambda_{2}} \\
& \leq c\left(\beta+\beta^{-2}\right)(\beta N)^{\frac{1-r}{2}}\left\|\partial_{v}^{r+1}\left(e^{-\frac{1}{2} \beta v} u\right)\right\|_{\omega_{r+1, \beta}^{2}, \Lambda_{2}} .
\end{aligned}
$$

2.2. Legendre approximations. We now turn to the Legendre approximation. For an integer $r \geq 0$, we define the space $H^{r}(I)$ and its norm $\|u\|_{r, I}$ as usual. We denote the inner product and norm of $L^{2}(I)$ by $(u, w)_{I}$ and $\|u\|_{I}$, respectively.

The Legendre polynomial of degree $m$ is defined by

$$
L_{m}(x)=\frac{(-1)^{m}}{2^{m} m !} \partial_{x}^{m}\left(1-x^{2}\right)^{m}
$$

The set of Legendre polynomials is a complete $L^{2}(I)$-orthogonal system.

Now, let $M$ be any positive integer. $\mathcal{P}_{M}(I)$ stands for the set of all polynomials of degree at most $M$. The orthogonal projection $P_{M, I}: L^{2}(I) \rightarrow \mathcal{P}_{M}(I)$ is defined by

$$
\left(P_{M, I} u-u, \phi\right)_{I}=0, \quad \forall \phi \in \mathcal{P}_{M}(I) .
$$

If $\left(1-x^{2}\right)^{\frac{r}{2}} \partial_{x}^{r} u \in L^{2}(I)$ and $r \geq 0$ is an integer, then (cf. page 389 of [14])

$$
\left\|P_{M, I} u-u\right\|_{I} \leq c M^{-r}\left\|\left(1-x^{2}\right)^{\frac{r}{2}} \partial_{x}^{r} u\right\|_{I} .
$$

With the aid of (2.14) and an argument as in 6, 12, we can improve the existing estimate for $\left\|\partial_{x}\left(P_{M, I} u-u\right)\right\|_{I}$. In other words, if $\partial_{x} u \in L^{2}(I),\left(1-x^{2}\right)^{\frac{r-1}{2}} \partial_{x}^{r} u \in$ $L^{2}(I)$ and $r \geq 1$ is an integer, then

$$
\left\|\partial_{x}\left(P_{M, I} u-u\right)\right\|_{I} \leq c M^{\frac{3}{2}-r}\left\|\left(1-x^{2}\right)^{\frac{r-1}{2}} \partial_{x}^{r} u\right\|_{I} .
$$

Next, let $\mathcal{P}_{M}^{0}(I)=\left\{\phi \mid \phi \in \mathcal{P}_{M}(I)\right.$ and $\left.\phi(-1)=\phi(1)=0\right\}$. The orthogonal projection $P_{M, I}^{1,0}: H_{0}^{1}(I) \rightarrow \mathcal{P}_{M}^{0}(I)$ is defined by

$$
\left(\partial_{x}\left(P_{M, I}^{1,0} u-u\right), \partial_{x} \phi\right)_{I}=0, \quad \forall \phi \in \mathcal{P}_{M}^{0}(I) .
$$

According to Theorem 3.4 of [19, we know that if $\partial_{x}^{s} u \in L^{2}(I),\left(1-x^{2}\right)^{\frac{r-1}{2}} \partial_{x}^{r} u \in$ $L^{2}(I), u(-1)=u(1)=0$, and $r \geq 1$ is an integer, then

$$
\left\|\partial_{x}^{s}\left(P_{M, I}^{1,0} u-u\right)\right\|_{I} \leq c M^{s-r}\left\|\left(1-x^{2}\right)^{\frac{r-1}{2}} \partial_{x}^{r} u\right\|_{I}, \quad s=0,1 .
$$


For the spectral method of hyperbolic equations, we have to consider a specific projection. To do this, let

${ }_{0} \mathcal{F}(I)=L^{2}(I) \cap\{u \mid$ there exists a finite trace of $u$ at $x=-1$, and $u(-1)=0\}$,

$$
{ }_{0} \mathcal{P}_{M}(I)=\left\{\phi \mid \phi \in \mathcal{P}_{M}(I) \text { and } \phi(-1)=0\right\} .
$$

The orthogonal projection ${ }_{0} P_{M, I}:{ }_{0} \mathcal{F}(I) \rightarrow{ }_{0} \mathcal{P}_{M}(I)$ is defined by

$$
\left({ }_{0} P_{M, I} u-u, \phi\right)_{I}=0, \quad \forall \phi \in{ }_{0} \mathcal{P}_{M}(I) .
$$

Lemma 2.2. If $u \in{ }_{0} \mathcal{F}(I),\left(1-x^{2}\right)^{\frac{r-1}{2}} \partial_{x}^{r} u \in L^{2}(I)$ and $r \geq 1$ is an integer, then

$$
\left\|{ }_{0} P_{M, I} u-u\right\|_{I} \leq c M^{-r}\left\|\left(1-x^{2}\right)^{\frac{r-1}{2}} \partial_{x}^{r} u\right\|_{I} .
$$

Proof. By the projection theorem,

$$
\left\|{ }_{0} P_{M, I} u-u\right\|_{I} \leq\|\phi-u\|_{I}, \quad \forall \phi \in{ }_{0} \mathcal{P}_{M}(I) .
$$

We follow the same line as in $[15$, to take

$$
\phi(x)=\int_{-1}^{x} P_{M-1, I} \partial_{\xi} u(\xi) d \xi \in{ }_{0} \mathcal{P}_{M}(I) .
$$

Due to the definition of $P_{M, I}$, we have that

$$
\phi(1)=\int_{I} P_{M-1, I} \partial_{\xi} u(\xi) d \xi=\int_{I} \partial_{\xi} u(\xi) d \xi=u(1) .
$$

Furthermore, let

$$
g(x)=\phi(x)-u(x), \quad G(x)=\int_{-1}^{x} g(\xi) d \xi .
$$

By integrating by parts, and using (2.14) and (2.18), we deduce that

$$
\begin{aligned}
\|\phi-u\|_{I}^{2} & =(\phi-u, g)_{I}=\left(\phi-u, \partial_{x} G\right)_{I} \\
& =-\left(\partial_{x}(\phi-u), G\right)_{I}=-\left(P_{M-1, I} \partial_{x} u-\partial_{x} u, G-P_{M-1, I} G\right)_{I} \\
& \leq c M^{-r}\left\|\left(1-x^{2}\right)^{\frac{r-1}{2}} \partial_{x}^{r} u\right\|_{I}\left\|\partial_{x} G\right\|_{I} \\
& \leq c M^{-r}\left\|\left(1-x^{2}\right)^{\frac{r-1}{2}} \partial_{x}^{r} u\right\|_{I}\|\phi-u\|_{I} .
\end{aligned}
$$

Then the desired result follows from the above and (2.17) immediately.

In order to obtain a better error estimate, we need other special projections. Let ${ }_{0} H^{1}(I)=H^{1}(I) \cap{ }_{0} \mathcal{F}(I)$. For any $u \in{ }_{0} H^{1}(I)$, we set $\tilde{u}(x)=u(x)-$ $\frac{1}{2}(x+1) u(1)$. Clearly, $\tilde{u} \in H_{0}^{1}(I)$. Then, we define the quasi-orthogonal projection ${ }_{0} P_{M, I}^{1}:{ }_{0} H^{1}(I) \rightarrow{ }_{0} \mathcal{P}_{M}(I)$ as

$$
{ }_{0} P_{M, I}^{1} u(x)=P_{M, I}^{1,0} \tilde{u}(x)+\frac{1}{2}(x+1) u(1) .
$$

Since ${ }_{0} P_{M, I}^{1} u(x)-u(x)=P_{M, I}^{1,0} \tilde{u}(x)-\tilde{u}(x)$, we use (2.16) to derive that if $u \in$ ${ }_{0} \mathcal{F}(I), \partial_{x}^{s} u \in L^{2}(I),\left(1-x^{2}\right)^{\frac{r-1}{2}} \partial_{x}^{r} u \in L^{2}(I)$ and $r \geq 2$ is an integer, then

$$
\begin{aligned}
\left\|\partial_{x}^{s}\left({ }_{0} P_{M, I}^{1} u-u\right)\right\|_{I} & =\left\|\partial_{x}^{s}\left(P_{M, I}^{1,0} \tilde{u}-\tilde{u}\right)\right\|_{I} \leq c M^{s-r}\left\|\left(1-x^{2}\right)^{\frac{r-1}{2}} \partial_{x}^{r} \tilde{u}\right\|_{I} \\
& =c M^{s-r}\left\|\left(1-x^{2}\right)^{\frac{r-1}{2}} \partial_{x}^{r} u\right\|_{I}, \quad s=0,1 .
\end{aligned}
$$

If $r=1$, then by using the trace theorem and the Poincaré inequality, we derive that $\left\|\partial_{x} \tilde{u}\right\|_{I} \leq\left\|\partial_{x} u\right\|_{I}+|u(1)| \leq c|| \partial_{x} u \|_{I}$. Thereby, the estimate (2.19) is also valid for $r \geq 1$. 
Remark 2.4. We may consider the generalized Jacobi orthogonal approximation $\bar{P}_{M, I}$ as in [17, by taking the polynomials $(x+1) J_{m-1}^{(0,1)}(x), 1 \leq m \leq M$, as the base functions. These polynomials are mutually orthogonal associated with the weight function $(1+x)^{-1}$. In this case, for any integers $0 \leq \mu \leq r$,

$$
\begin{aligned}
\int_{I}(1-x)^{\mu}(1 & +x)^{\mu-1}\left(\partial_{x}^{\mu}\left(\bar{P}_{M, I} u(x)-u(x)\right)\right)^{2} d x \\
& \leq c M^{\mu-r} \int_{I}(1-x)^{r}(1+x)^{r-1}\left(\partial_{x}^{r} u(x)\right)^{2} d x .
\end{aligned}
$$

But in this paper, we need to estimate the $H^{1}(I)$-norm of the difference between $u(x)$ and its orthogonal projection. Therefore, we cannot use the above result with $\mu=1$, by which we could not measure the approximation error precisely as $x \rightarrow 1$. This is the reason why we derived the upper-bound of $\left\|\partial_{x}\left({ }_{0} P_{M, I}^{1} u-u\right)\right\|_{I}$.

In this work, we shall use other kinds of approximations. Let

$$
\begin{gathered}
{ }^{0} \mathcal{F}(I)=L^{2}(I) \cap\{u \mid \text { there exists a finite trace of } u \text { at } x=1, \text { and } u(1)=0\}, \\
{ }^{0} \mathcal{P}_{M}(I)=\left\{\phi \mid \phi \in \mathcal{P}_{M}(I) \text { and } \phi(1)=0\right\} .
\end{gathered}
$$

The orthogonal projection ${ }^{0} P_{M, I}:{ }^{0} \mathcal{F}(I) \rightarrow{ }^{0} \mathcal{P}_{M}(I)$ is defined by

$$
\left({ }^{0} P_{M, I} u-u, \phi\right)_{I}=0, \quad \forall \phi \in{ }^{0} \mathcal{P}_{M}(I) .
$$

If $u \in{ }^{0} \mathcal{F}(I),\left(1-x^{2}\right)^{\frac{r-1}{2}} \partial_{x}^{r} u \in L^{2}(I)$ and $r \geq 1$ is an integer, then

$$
\left\|{ }^{0} P_{M, I} u-u\right\|_{I} \leq c M^{-r}\left\|\left(1-x^{2}\right)^{\frac{r-1}{2}} \partial_{x}^{r} u\right\|_{I} .
$$

On the other hand, let ${ }^{0} H^{1}(I)=H^{1}(I) \cap{ }^{0} \mathcal{F}(I)$. For any $u \in{ }^{0} H^{1}(I)$, we set $\tilde{u}(x)=$ $u(x)-\frac{1}{2}(1-x) u(-1) \in H_{0}^{1}(I)$. Then, we define the quasi-orthogonal projection ${ }^{0} P_{M, I}^{1}:{ }^{0} H^{1}(I) \rightarrow{ }^{0} \mathcal{P}_{M}(I)$ as

$$
{ }^{0} P_{M, I}^{1} u(x)=P_{M, I}^{1,0} \tilde{u}(x)+\frac{1}{2}(1-x) u(-1) .
$$

By an argument similar to the derivation of (2.19), we observe that if $u \in{ }^{0} \mathcal{F}(I), \partial_{x}^{s} u$ $\in L^{2}(I),\left(1-x^{2}\right)^{\frac{r-1}{2}} \partial_{x}^{r} u \in L^{2}(I)$ and $r \geq 1$ is an integer, then

$$
\left\|\partial_{x}^{s}\left({ }^{0} P_{M, I}^{1} u-u\right)\right\|_{I} \leq c M^{s-r}\left\|\left(1-x^{2}\right)^{\frac{r-1}{2}} \partial_{x}^{r} u\right\|_{I}, \quad s=0,1 .
$$

2.3. Mixed generalized Laguerre-Legendre approximations. We now investigate the mixed generalized Laguerre-Legendre approximations. Let $\Omega_{1}=I \times \Lambda_{1}$ and $\chi(x, v)$ be a certain weight function. We define the space $L_{\chi}^{2}\left(\Omega_{1}\right)$ as usual, with the following inner product and norm:

$$
(u, w)_{\chi, \Omega_{1}}=\iint_{\Omega_{1}} u(x, v) w(x, v) \chi(x, v) d x d v, \quad\|u\|_{\chi, \Omega_{1}}=(u, u)_{\chi, \Omega_{1}}^{\frac{1}{2}} .
$$

We omit the subscript $\chi$ in the notation when $\chi(x, v) \equiv 1$.

Now, let ${ }_{0} \mathcal{F}_{v^{\alpha}}\left(\Omega_{1}\right)=L_{v^{\alpha}}^{2}\left(\Lambda_{1} ;{ }_{0} \mathcal{F}(I)\right)$ and ${ }_{0} V_{M, N, \beta}\left(\Omega_{1}\right)={ }_{0} \mathcal{P}_{M}(I) \otimes \mathcal{Q}_{N, \beta}\left(\Lambda_{1}\right)$. The orthogonal projection $P_{M, N, \alpha, \beta, \Omega_{1}} u=\left(\tilde{P}_{N, \alpha, \beta, \Lambda_{1}}{ }_{0} P_{M, I}\right) u$. In order to estimate $\left\|P_{M, N, \alpha, \beta, \Omega_{1}} u-u\right\|_{v^{\alpha}, \Omega_{1}}$, we use the following notation with integers $q, r \geq 0$ :

$$
\mathbb{A}_{\omega_{\alpha, \beta}^{1}, \Omega_{1}}^{q, r}(u)=\int_{I}\left\|\partial_{v}^{r}\left(e^{\frac{1}{2} \beta v} u\right)\right\|_{\omega_{r+\alpha, \beta}^{1}, \Lambda_{1}}^{2} d x+\int_{\Lambda_{1}} v^{\alpha}\left\|\left(1-x^{2}\right)^{\frac{q-1}{2}} \partial_{x}^{q} u\right\|_{I}^{2} d v .
$$


Theorem 2.1. For any $u \in{ }_{0} \mathcal{F}_{v^{\alpha}}\left(\Omega_{1}\right)$ and integers $q, r \geq 0$,

$$
\left\|P_{M, N, \alpha, \beta, \Omega_{1}} u-u\right\|_{v^{\alpha}, \Omega_{1}}^{2} \leq c\left(M^{-2 q}+(\beta N)^{-r}\right) \mathbb{A}_{\omega_{\alpha, \beta}^{1}, \Omega_{1}}^{q, r}(u),
$$

provided that $\mathbb{A}_{\omega_{\alpha, \beta}^{\prime}, \Omega_{1}}^{q, r}(u)$ is finite.

Proof. By virtue of (2.4) and Lemma 2.2,

$$
\begin{aligned}
& \left\|P_{M, N, \alpha, \beta, \Omega_{1}} u-u\right\|_{v^{\alpha}, \Omega_{1}}^{2} \\
& \leq 2\left\|_{0} P_{M, I}\left(\tilde{P}_{N, \alpha, \beta, \Lambda_{1}} u\right)-\tilde{P}_{N, \alpha, \beta, \Lambda_{1}} u\right\|_{v^{\alpha}, \Omega_{1}}^{2}+2\left\|\tilde{P}_{N, \alpha, \beta, \Lambda_{1}} u-u\right\|_{v^{\alpha}, \Omega_{1}}^{2} \\
& \leq c M^{-2 q} \int_{\Lambda_{1}} v^{\alpha}\left\|\left(1-x^{2}\right)^{\frac{q-1}{2}} \partial_{x}^{q} \tilde{P}_{N, \alpha, \beta, \Lambda_{1}} u\right\|_{I}^{2} d v \\
& \quad+c(\beta N)^{-r} \int_{I}\left\|\partial_{v}^{r}\left(e^{\frac{1}{2} \beta v} u\right)\right\|_{\omega_{r+\alpha, \beta}^{1}, \Lambda_{1}}^{2} d x .
\end{aligned}
$$

Moreover, thanks to (2.4) with $r=0$, we have

$$
\int_{\Lambda_{1}} v^{\alpha}\left\|\left(1-x^{2}\right)^{\frac{q-1}{2}} \partial_{x}^{q} \tilde{P}_{N, \alpha, \beta, \Lambda_{1}} u\right\|_{I}^{2} d v \leq c \int_{\Lambda_{1}} v^{\alpha}\left\|\left(1-x^{2}\right)^{\frac{q-1}{2}} \partial_{x}^{q} u\right\|_{I}^{2} d v .
$$

Then the desired result follows immediately.

For spectral methods with domain decomposition, we should consider the orthogonal projection ${ }_{*} P_{M, N, \beta, \Omega_{1}} u=\left({ }_{*} \tilde{P}_{N, \beta, \Lambda_{1}} \cdot{ }_{0} P_{M, I}\right) u$. Following the same line as in the proof of Theorem 2.1, we use (2.5) and Lemma 2.2 to obtain that

$$
\left\|{ }_{*} P_{M, N, \beta, \Omega_{1}} u-u\right\|_{\Omega_{1}}^{2} \leq c\left(M^{-2 q}+(\beta N)^{-r}\right)_{*} \mathbb{A}_{\omega_{0, \beta}^{1}, \Omega_{1}}^{q, r}(u),
$$

where

$$
\begin{aligned}
* \mathbb{A}_{\omega_{0, \beta}^{1}, \Omega_{1}}^{q, r}(u) & =\int_{I}\left\|\partial_{v}^{r+1}\left(e^{\frac{1}{2} \beta v} u\right)\right\|_{\omega_{r, \beta}^{1}, \Lambda_{1}}^{2} d x \\
& +\int_{\Lambda_{1}}\left(\left\|\left(1-x^{2}\right)^{\frac{q-1}{2}} \partial_{x}^{q} u\right\|_{I}^{2}+\beta^{-2} e^{-\beta v}\left\|\left(1-x^{2}\right)^{\frac{q-1}{2}} \partial_{x}^{q} \partial_{v}\left(e^{\frac{1}{2} \beta v} u\right)\right\|_{I}^{2}\right) d v .
\end{aligned}
$$

In the numerical analysis of the composite spectral method for the FokkerPlanck equation, we need a specific projection. For this purpose, let ${ }_{0} \mathcal{F}_{1, v^{2}+1}^{1}\left(\Omega_{1}\right)=H_{1, v^{2}+1}^{1}\left(\Lambda_{1} ;{ }_{0} \mathcal{F}(I)\right)$ equipped with the norm $\|u\|_{1,1, v^{2}+1, \Omega_{1}}=$ $\left(\left\|\partial_{v} u\right\|_{\Omega_{1}}^{2}+\|u\|_{v^{2}+1, \Omega_{1}}^{2}\right)^{\frac{1}{2}}$.

The quasi-orthogonal projection $P_{M, N, \beta, \Omega_{1}}^{1} u=\left(P_{N, \beta, \Lambda_{1}}^{1}{ }_{0} P_{M, I}^{1}\right) u$. For describing approximation results, we use the following notation with integers $q, r \geq 1$ :

$$
\begin{aligned}
& \mathbb{B}_{\beta, \Omega_{1}}^{q, r}(u) \\
& =\left(\beta+\beta^{-2}\right)^{2} \int_{I}\left(\left\|\partial_{v}^{r+1}\left(e^{\frac{1}{2} \beta v} u\right)\right\|_{\omega_{r+1, \beta}^{1}, \Lambda_{1}}^{2}+\left(1-x^{2}\right)^{q-1}\left\|\partial_{v}^{2}\left(e^{\frac{1}{2} \beta v} \partial_{x}^{q} u\right)\right\|_{\omega_{2, \beta}^{1}, \Lambda_{1}}^{2}\right) d x \\
& +\int_{\Lambda_{1}}\left(\left\|\left(1-x^{2}\right)^{\frac{q-1}{2}} \partial_{x}^{q} \partial_{v} u\right\|_{I}^{2}+\left(v^{2}+1\right)\left\|\left(1-x^{2}\right)^{\frac{q-1}{2}} \partial_{x}^{q} u\right\|_{I}^{2}\right) d v .
\end{aligned}
$$

Theorem 2.2. For any $u \in{ }_{0} \mathcal{F}_{1, v^{2}+1}^{1}\left(\Omega_{1}\right)$ and integers $q, r \geq 1$,

$$
\begin{gathered}
\left\|\partial_{v}\left(P_{M, N, \beta, \Omega_{1}}^{1} u-u\right)\right\|_{\Omega_{1}}^{2}+\left\|P_{M, N, \beta, \Omega_{1}}^{1} u-u\right\|_{v^{2}+1, \Omega_{1}}^{2} \\
\leq c\left(M^{-2 q}+(\beta N)^{1-r}\right) \mathbb{B}_{\beta, \Omega_{1}}^{q, r}(u),
\end{gathered}
$$

provided that $\mathbb{B}_{\beta, \Omega_{1}}^{q, r}(u)$ is finite. 
Proof. Since $P_{M, N, \beta, \Omega_{1}}^{1} u=P_{N, \beta, \Lambda_{1}}^{1}\left({ }_{0} P_{M, I}^{1} u\right)$, we have that

$$
\left\|\partial_{v}\left(P_{M, N, \beta, \Omega_{1}}^{1} u-u\right)\right\|_{\Omega_{1}}^{2}+\left\|P_{M, N, \beta, \Omega_{1}}^{1} u-u\right\|_{v^{2}+1, \Omega_{1}}^{2} \leq F_{1}(u)+F_{2}(u)+F_{3}(u),
$$

where

$$
\begin{aligned}
& F_{1}(u)=2\left\|\partial_{v}\left(P_{N, \beta, \Lambda_{1}}^{1} u-u\right)\right\|_{\Omega_{1}}^{2}+2\left\|P_{N, \beta, \Lambda_{1}}^{1} u-u\right\|_{v^{2}+1, \Omega_{1}}^{2}, \\
& F_{2}(u)=2\left\|\partial_{v}\left({ }_{0} P_{M, I}^{1}\left(P_{N, \beta, \Lambda_{1}}^{1} u\right)-P_{N, \beta, \Lambda_{1}}^{1} u\right)\right\|_{\Omega_{1}}^{2} \\
& F_{3}(u)=2\left\|P_{M, I}^{1}\left(P_{N, \beta, \Lambda_{1}}^{1} u\right)-P_{N, \beta, \Lambda_{1}}^{1} u\right\|_{v^{2}+1, \Omega_{1}}^{2} .
\end{aligned}
$$

Due to Lemma 2.1,

$$
F_{1}(u) \leq c\left(\beta+\beta^{-2}\right)^{2}(\beta N)^{1-r} \int_{I}\left\|\partial_{v}^{r+1}\left(e^{\frac{1}{2} \beta v} u\right)\right\|_{\omega_{r+1, \beta}^{1}, \Lambda_{1}}^{2} d x
$$

Next, by using Lemma 2.1 with $r=1$ and (2.19) with $s=0$, we obtain that

$$
\begin{aligned}
F_{2}(u) & \leq c M^{-2 q} \int_{\Lambda_{1}}\left\|\left(1-x^{2}\right)^{\frac{q-1}{2}} \partial_{x}^{q} \partial_{v} P_{N, \beta, \Lambda_{1}}^{1} u\right\|_{I}^{2} d v \\
& \leq c M^{-2 q} \int_{\Lambda_{1}}\left\|\left(1-x^{2}\right)^{\frac{q-1}{2}} \partial_{x}^{q} \partial_{v} u\right\|_{I}^{2} d v \\
& +c\left(\beta+\beta^{-2}\right)^{2} M^{-2 q} \int_{I}\left(1-x^{2}\right)^{q-1}\left\|\partial_{v}^{2}\left(e^{\frac{1}{2} \beta v} \partial_{x}^{q} u\right)\right\|_{\omega_{2, \beta}^{1}, \Lambda_{1}}^{2} d x .
\end{aligned}
$$

Similarly,

$$
\begin{aligned}
F_{3}(u) & \leq c M^{-2 q} \int_{\Lambda_{1}}\left(v^{2}+1\right)\left\|\left(1-x^{2}\right)^{\frac{q-1}{2}} \partial_{x}^{q} P_{N, \beta, \Lambda_{1}}^{1} u\right\|_{I}^{2} d v \\
& \leq c M^{-2 q} \int_{\Lambda_{1}}\left(v^{2}+1\right)\left\|\left(1-x^{2}\right)^{\frac{q-1}{2}} \partial_{x}^{q} u\right\|_{I}^{2} d v \\
& +c\left(\beta+\beta^{-2}\right)^{2} M^{-2 q} \int_{I}\left(1-x^{2}\right)^{q-1}\left\|\partial_{v}^{2}\left(e^{\frac{1}{2} \beta v} \partial_{x}^{q} u\right)\right\|_{\omega_{2, \beta}^{1}, \Lambda_{1}}^{2} d x .
\end{aligned}
$$

A combination of previous estimates leads to the desired result.

We now turn to the approximations on the domain $\Omega_{2}=I \times \Lambda_{2}$. We define the space $L_{\chi}^{2}\left(\Omega_{2}\right)$ as usual, with the inner product $(u, w)_{\chi, \Omega_{2}}$ and the norm $\|u\|_{\chi, \Omega_{2}}$. Also, we set ${ }^{0} \mathcal{F}_{(-v)^{\alpha}}\left(\Omega_{2}\right)=L_{(-v)^{\alpha}}^{2}\left(\Lambda_{2} ;{ }^{0} \mathcal{F}(I)\right)$ and ${ }^{0} V_{M, N, \beta}\left(\Omega_{2}\right)={ }^{0} \mathcal{P}_{M}(I) \otimes$ $\mathcal{Q}_{N, \beta}\left(\Lambda_{2}\right)$.

The orthogonal projection $P_{M, N, \alpha, \beta, \Omega_{2}} u=\left(\tilde{P}_{N, \alpha, \beta, \Lambda_{2}} \cdot{ }^{0} P_{M, I}\right) u$. Let

$$
\mathbb{A}_{\omega_{\alpha, \beta}^{2}, \Omega_{2}}^{q, r}(u)=\int_{I}\left\|\partial_{v}^{r}\left(e^{-\frac{1}{2} \beta v} u\right)\right\|_{\omega_{r+\alpha, \beta}^{2}, \Lambda_{2}}^{2} d x+\int_{\Lambda_{2}}(-v)^{\alpha}\left\|\left(1-x^{2}\right)^{\frac{q-1}{2}} \partial_{x}^{q} u\right\|_{I}^{2} d v .
$$

Following the same line as in the proof of Theorem 2.1, we show that for integers $q, r \geq 0$,

$$
\left\|P_{M, N, \alpha, \beta, \Omega_{2}} u-u\right\|_{(-v)^{\alpha}, \Omega_{2}}^{2} \leq c\left(M^{-2 q}+(\beta N)^{-r}\right) \mathbb{A}_{\omega_{\alpha, \beta}^{2}, \Omega_{2}}^{q, r}(u),
$$

provided that $\mathbb{A}_{\omega_{\alpha, \beta}^{2}, \Omega_{2}}^{q, r}(u)$ is finite.

We also introduce the quasi-orthogonal projection ${ }_{*} P_{M, N, \beta, \Omega_{2}} u=\left({ }_{*} \tilde{P}_{N, \beta, \Lambda_{2}}\right.$. $\left.{ }^{0} P_{M, I}\right) u$. Following the same line as in the proof of Theorem 2.1, we use (2.12) and (2.20) to obtain that

$$
\left\|{ }_{*} P_{M, N, \beta, \Omega_{2}} u-u\right\|_{\Omega_{2}}^{2} \leq c\left(M^{-2 q}+(\beta N)^{-r}\right)_{*} \mathbb{A}_{\omega_{0, \beta}^{2}, \Omega_{2}}^{q, r}(u),
$$


where

$$
\begin{aligned}
* \mathbb{A}_{\omega_{0, \beta}^{2}, \Omega_{2}}^{q, r}(u) & =\int_{I}\left\|\partial_{v}^{r+1}\left(e^{-\frac{1}{2} \beta v} u\right)\right\|_{\omega_{r, \beta}^{2}, \Lambda_{2}}^{2} d x \\
& +\int_{\Lambda_{2}}\left(\left\|\left(1-x^{2}\right)^{\frac{q-1}{2}} \partial_{x}^{q} u\right\|_{I}^{2}+\beta^{-2} e^{\beta v}\left\|\left(1-x^{2}\right)^{\frac{q-1}{2}} \partial_{x}^{q} \partial_{v}\left(e^{-\frac{1}{2} \beta v} u\right)\right\|_{I}^{2}\right) d v .
\end{aligned}
$$

Next, let ${ }^{0} \mathcal{F}_{1, v^{2}+1}^{1}\left(\Omega_{2}\right)=H_{1, v^{2}+1}^{1}\left(\Lambda_{2},{ }^{0} \mathcal{F}(I)\right)$, equipped with the norm $\|u\|_{1,1, v^{2}+1, \Omega_{2}}=\left(\left\|\partial_{v} u\right\|_{\Omega_{2}}^{2}+\|u\|_{v^{2}+1, \Omega_{2}}^{2}\right)^{\frac{1}{2}}$.

The quasi-orthogonal projection $P_{M, N, \beta, \Omega_{2}}^{1} u=\left(P_{N, \beta, \Lambda_{2}}^{1} \cdot{ }^{0} P_{M, I}^{1}\right) u$. Let

$$
\begin{aligned}
& \mathbb{B}_{\beta, \Omega_{2}}^{q, r}(u) \\
& =\left(\beta+\beta^{-2}\right)^{2} \int_{I}\left(\left\|\partial_{v}^{r+1}\left(e^{-\frac{1}{2} \beta v} u\right)\right\|_{\omega_{r+1, \beta}^{2}, \Lambda_{2}}^{2}+\left(1-x^{2}\right)^{q-1}\left\|\partial_{v}^{2}\left(e^{-\frac{1}{2} \beta v} \partial_{x}^{q} u\right)\right\|_{\omega_{2, \beta}^{2}, \Lambda_{2}}^{2}\right) d x \\
& +\int_{\Lambda_{2}}\left(\left\|\left(1-x^{2}\right)^{\frac{q-1}{2}} \partial_{x}^{q} \partial_{v} u\right\|_{I}^{2}+\left(v^{2}+1\right)\left\|\left(1-x^{2}\right)^{\frac{q-1}{2}} \partial_{x}^{q} u\right\|_{I}^{2}\right) d v .
\end{aligned}
$$

Like Theorem 2.2, we have that for $u \in{ }^{0} \mathcal{F}_{1, v^{2}+1}^{1}\left(\Omega_{2}\right)$ and integers $q, r \geq 1$,

$$
\begin{aligned}
& \left\|\partial_{v}\left(P_{M, N, \beta, \Omega_{2}}^{1} u-u\right)\right\|_{\Omega_{2}}^{2}+\left\|P_{M, N, \beta, \Omega_{2}}^{1} u-u\right\|_{v^{2}+1, \Omega_{2}}^{2} \\
& \leq c\left(M^{-2 q}+(\beta N)^{1-r}\right) \mathbb{B}_{\beta, \Omega_{2}}^{q, r}(u),
\end{aligned}
$$

provided that $\mathbb{B}_{\beta, \Omega_{2}}^{q, r}(u)$ is finite.

2.4. Composite approximations. We now consider several composite generalized Laguerre-Legendre approximations, which will be used in the next section. The space $L_{\chi}^{2}(\Omega)$ is defined as usual, with the inner product $(u, w)_{\chi, \Omega}$ and the norm $\|u\|_{\chi, \Omega}$. We omit the subscript $\chi$ in the notation when $\chi(x, v) \equiv 1$. Let

$$
\begin{gathered}
\mathbb{M}(\Omega)=\left\{u\left|u \in L_{v^{2}+1}^{2}(\Omega), \partial_{x} u, \partial_{v} u \in L^{2}(\Omega), u\right|_{\Gamma_{1}}=0 \text { and }\left.u\right|_{\Gamma_{2}} \in L_{|v|}^{2}\left(\Gamma_{2}\right)\right\}, \\
V_{M, N, \beta}(\Omega)=\mathbb{M}(\Omega) \cap\left\{\phi|\phi|_{\Omega_{1}} \in \mathcal{Q}_{N, \beta, \Lambda_{1}} \otimes{ }_{0} \mathcal{P}_{M}(I) ;\left.\phi\right|_{\Omega_{2}} \in \mathcal{Q}_{N, \beta, \Lambda_{2}} \otimes{ }^{0} \mathcal{P}_{M}(I)\right\} .
\end{gathered}
$$

The composite approximations are defined as

$$
\begin{gathered}
\left.P_{M, N, \beta, \Omega} u(x, v)\right|_{\Omega_{j}}=P_{M, N, 0, \beta, \Omega_{j}} u(x, v),\left.\quad{ }_{*} P_{M, N, \beta, \Omega} u(x, v)\right|_{\Omega_{j}}={ }_{*} P_{M, N, \beta, \Omega_{j}} u(x, v), \\
\left.P_{M, N, \beta, \Omega}^{1} u(x, v)\right|_{\Omega_{j}}=P_{M, N, \beta, \Omega_{j}}^{1} u(x, v), \quad j=1,2 .
\end{gathered}
$$

It is noted that ${ }_{*} P_{M, N, \beta, \Omega} u, P_{M, N, \beta, \Omega}^{1} u \in V_{M, N, \beta}(\Omega)$. But $P_{M, N, \beta, \Omega} u$ has a jump at $v=0$.

For a description of approximation errors, we introduce the following notation:

$$
\mathbb{A}_{\beta, \Omega}^{q, r}(u)=\sum_{j=1}^{2} \mathbb{A}_{\omega_{0, \beta}^{j}, \Omega_{j}}^{q, r}(u), \quad{ }_{*} \mathbb{A}_{\beta, \Omega}^{q, r}(u)=\sum_{j=1}^{2}{ }_{*} \mathbb{A}_{\omega_{0, \beta}^{j}, \Omega_{j}}^{q, r}(u), \quad \mathbb{B}_{\beta, \Omega}^{q, r}(u)=\sum_{j=1}^{2} \mathbb{B}_{\beta, \Omega_{j}}^{q, r}(u) .
$$

We can use (2.22)-(2.27) to derive that

$$
\begin{gathered}
\left\|P_{M, N, \beta, \Omega} u-u\right\|_{\Omega}^{2} \leq c\left(M^{-2 q}+(\beta N)^{-r}\right) \mathbb{A}_{\beta, \Omega}^{q, r}(u), \\
\left\|_{*} P_{M, N, \beta, \Omega} u-u\right\|_{\Omega}^{2} \leq c\left(M^{-2 q}+(\beta N)^{-r}\right)_{*} \mathbb{A}_{\beta, \Omega}^{q, r}(u), \\
\left\|\partial_{v}\left(P_{M, N, \beta, \Omega}^{1} u-u\right)\right\|_{\Omega}^{2}+\left\|P_{M, N, \beta, \Omega}^{1} u-u\right\|_{v^{2}+1, \Omega}^{2} \\
\leq c\left(M^{-2 q}+(\beta N)^{1-r}\right) \mathbb{B}_{\beta, \Omega}^{q, r}(u) .
\end{gathered}
$$
by

Another useful composite projection ${ }_{* *} P_{M, N, \beta, \Omega}: L^{2}(\Omega) \rightarrow V_{M, N, \beta}(\Omega)$ is defined

$$
\left({ }_{* *} P_{M, N, \beta, \Omega} u-u, \phi\right)_{\Omega}=0, \quad \forall \phi \in V_{M, N, \beta}(\Omega) .
$$


By the projection theorem,

$$
\|\|_{* *} P_{M, N, \beta, \Omega} u-u\left\|_{\Omega} \leq\right\|_{*} P_{M, N, \beta, \Omega} u-u \|_{\Omega} \leq c\left(M^{-2 q}+(\beta N)^{-r}\right)_{*} \mathbb{A}_{\beta, \Omega}^{q, r}(u) .
$$

\section{Composite Spectral SCheme For FokKer-Planck EQUation}

In this section, we propose the composite spectral scheme for the Fokker-Planck equation in an infinite channel, with the convergence analysis. We also describe the implementation and present some numerical results.

\subsection{Composite spectral scheme. Let}

$$
\tilde{\mathbb{M}}(\Omega)=\left\{u\left|u \in L_{v^{2}+1}^{2}(\Omega), \partial_{v} u \in L^{2}(\Omega), u\right|_{\Gamma_{1}}=0 \text { and }\left.u\right|_{\Gamma_{2}} \in L_{|v|}^{2}\left(\Gamma_{2}\right)\right\} .
$$

A weak formulation of (1.1) is to seek a solution $W \in L^{\infty}\left(0, T ; L^{2}(\Omega)\right) \quad \cap$ $L^{2}(0, T ; \tilde{\mathbb{M}}(\Omega))$ such that

$$
\left\{\begin{array}{l}
\left(\partial_{t} W(t), u\right)_{\Omega}-\left(v W(t), \partial_{x} u\right)_{\Omega}+\beta_{0}\left(v W(t), \partial_{v} u\right)_{\Omega}+\gamma\left(x \partial_{v} W(t), u\right)_{\Omega} \\
\quad+\beta_{0} \mu\left(\partial_{v} W(t), \partial_{v} u\right)_{\Omega}+\int_{\Gamma_{2}}|v| W(\cdot, v, t) u(\cdot, v) d v=0, \quad \forall u \in \mathbb{M}, 0<t \leq T, \\
W(0)=W_{0} .
\end{array}\right.
$$

Following the same line as in the derivation of the main result of [26, it can be proved that if $W_{0} \in L_{v^{2}+1}^{2}(\Omega)$, then (3.1) has a unique solution $W \in L^{\infty}\left(0, T ; L^{2}(\Omega)\right)$ $\cap L^{2}(0, T ; \tilde{\mathbb{M}}(\Omega))$. If, in addition, $\partial_{x} W_{0} \in L^{2}(\Omega)$, then $\partial_{x} W \in L^{\infty}\left(0, T ; L^{2}(\Omega)\right)$. We focus on the smooth solution of (3.1), i.e., $\partial_{x} W \in L^{2}\left(0, T ; L^{2}(\Omega)\right)$. In this case, an alternative form of (3.1) is to find $W \in L^{\infty}\left(0, T ; L^{2}(\Omega)\right) \cap L^{2}(0, T ; \mathbb{M}(\Omega))$ such that

$$
\left\{\begin{array}{l}
\left(\partial_{t} W(t), u\right)_{\Omega}+\left(v \partial_{x} W(t), u\right)_{\Omega}+\beta_{0}\left(v W(t), \partial_{v} u\right)_{\Omega}+\gamma\left(x \partial_{v} W(t), u\right)_{\Omega} \\
\quad+\beta_{0} \mu\left(\partial_{v} W(t), \partial_{v} u\right)_{\Omega}=0, \quad \forall u \in \mathbb{M}, 0<t \leq T \\
W(0)=W_{0}
\end{array}\right.
$$

We now design the composite generalized Laguerre-Legendre spectral scheme for (3.2). It is to find $w_{M, N}(t) \in V_{M, N, \beta}(\Omega)$ for all $0 \leq t \leq T$ such that

$$
\left\{\begin{array}{c}
\left(\partial_{t} w_{M, N}(t), \phi\right)_{\Omega}+\left(v \partial_{x} w_{M, N}(t), \phi\right)_{\Omega}+\beta_{0}\left(v w_{M, N}(t), \partial_{v} \phi\right)_{\Omega} \\
+\gamma\left(x \partial_{v} w_{M, N}(t), \phi\right)_{\Omega}+\beta_{0} \mu\left(\partial_{v} w_{M, N}(t), \partial_{v} \phi\right)_{\Omega}=0 \\
\quad \forall \phi \in V_{M, N, \beta}(\Omega), 0<t \leq T \\
w_{M, N}(0)={ }_{*} P_{M, N, \beta, \Omega} W_{0}, \quad \text { or }{ }_{* *} P_{M, N, \beta, \Omega} W_{0} .
\end{array}\right.
$$

3.2. Error analysis. We next deal with the convergence of scheme (3.3). Let $W_{M, N}=P_{M, N, \beta, \Omega}^{1} W$. We have from (3.2) that

$$
\left\{\begin{array}{c}
\left(\partial_{t} W_{M, N}(t), \phi\right)_{\Omega}+\left(v \partial_{x} W_{M, N}(t), \phi\right)_{\Omega}+\beta_{0}\left(v W_{M, N}(t), \partial_{v} \phi\right)_{\Omega} \\
+\gamma\left(x \partial_{v} W_{M, N}(t), \phi\right)_{\Omega}+\beta_{0} \mu\left(\partial_{v} W_{M, N}(t), \partial_{v} \phi\right)_{\Omega}+\sum_{j=1}^{5} G_{j}(t, \phi)=0, \\
\quad \forall \phi \in V_{M, N, \beta}(\Omega), 0<t \leq T, \\
W_{M, N}(0)=P_{M, N, \beta, \Omega}^{1} W_{0},
\end{array}\right.
$$


where

$$
\begin{aligned}
& G_{1}(t, \phi)=\left(\partial_{t} W(t)-\partial_{t} W_{M, N}(t), \phi\right)_{\Omega}, \\
& G_{2}(t, \phi)=\left(v \partial_{x} W(t)-v \partial_{x} W_{M, N}(t), \phi\right)_{\Omega}, \\
& G_{3}(t, \phi)=\beta_{0}\left(v W(t)-v W_{M, N}(t), \partial_{v} \phi\right)_{\Omega}, \\
& G_{4}(t, \phi)=\gamma\left(x \partial_{v} W(t)-x \partial_{v} W_{M, N}(t), \phi\right)_{\Omega}, \\
& G_{5}(t, \phi)=\beta_{0} \mu\left(\partial_{v} W(t)-\partial_{v} W_{M, N}(t), \partial_{v} \phi\right)_{\Omega} .
\end{aligned}
$$

Setting $\tilde{W}_{M, N}=w_{M, N}-W_{M, N}$ and subtracting (3.4) from (3.3), we obtain that (3.5)

$$
\left\{\begin{array}{r}
\left(\partial_{t} \tilde{W}_{M, N}(t), \phi\right)_{\Omega}+\left(v \partial_{x} \tilde{W}_{M, N}(t), \phi\right)_{\Omega}+\beta_{0}\left(v \tilde{W}_{M, N}(t), \partial_{v} \phi\right)_{\Omega} \\
+\gamma\left(x \partial_{v} \tilde{W}_{M, N}(t), \phi\right)_{\Omega}+\beta_{0} \mu\left(\partial_{v} \tilde{W}_{M, N}(t), \partial_{v} \phi\right)_{\Omega}=\sum_{j=1}^{5} G_{j}(t, \phi), \\
\quad \forall \phi \in V_{M, N, \beta}(\Omega), 0<t \leq T, \\
\tilde{W}_{M, N}(0)={ }_{*} P_{M, N, \beta, \Omega} W_{0}-P_{M, N, \beta, \Omega}^{1} W_{0}, \quad \text { or }{ }_{* *} P_{M, N, \beta, \Omega} W_{0}-P_{M, N, \beta, \Omega}^{1} W_{0} .
\end{array}\right.
$$

By integration by parts, we observe that

$$
\begin{aligned}
2\left(v \partial_{x} \tilde{W}_{M, N}(t), \tilde{W}_{M, N}(t)\right)_{\Omega} & =\int_{\Gamma_{2}}|v| \tilde{W}_{M, N}^{2}(t) d v, \\
2 \beta_{0}\left(v \tilde{W}_{M, N}(t), \partial_{v} \tilde{W}_{M, N}(t)\right)_{\Omega} & =-\beta_{0}\left\|\tilde{W}_{M, N}(t)\right\|_{\Omega}^{2} .
\end{aligned}
$$

Clearly,

$$
2 \gamma\left|\left(x \partial_{v} \tilde{W}_{M, N}(t), \tilde{W}_{M, N}(t)\right)_{\Omega}\right| \leq \frac{1}{2} \beta_{0} \mu\left\|\partial_{v} \tilde{W}_{M, N}(t)\right\|_{\Omega}^{2}+\frac{2 \gamma^{2}}{\beta_{0} \mu}\left\|\tilde{W}_{M, N}(t)\right\|_{\Omega}^{2} .
$$

Hence, by taking $\phi=2 \tilde{W}_{M, N}$ in (3.5) and using (3.6)-(3.8), we obtain that

$$
\begin{gathered}
\partial_{t}\left\|\tilde{W}_{M, N}(t)\right\|_{\Omega}^{2}+\frac{3}{2} \beta_{0} \mu\left\|\partial_{v} \tilde{W}_{M, N}(t)\right\|_{\Omega}^{2}+\int_{\Gamma_{2}}|v| \tilde{W}_{M, N}^{2}(t) d v \\
\leq 2 \sum_{j=1}^{5} G_{j}\left(t, \tilde{W}_{M, N}(t)\right)+\left(\beta_{0}+\frac{2 \gamma^{2}}{\beta_{0} \mu}\right)\left\|\tilde{W}_{M, N}(t)\right\|_{\Omega}^{2} .
\end{gathered}
$$

Therefore, it remains to estimate the terms $\left|G_{j}\left(t, \tilde{W}_{M, N}(s)\right)\right|, 1 \leq j \leq 5$.

We use the Cauchy inequality and (2.30) to verify that for integers $q, r \geq 1$,

$$
\begin{aligned}
& 2\left|G_{1}\left(t, \tilde{W}_{M, N}(t)\right)\right| \leq\left\|\partial_{t}\left(W(t)-W_{M, N}(t)\right)\right\|_{\Omega}^{2}+\left\|\tilde{W}_{M, N}(t)\right\|_{\Omega}^{2} \\
& \quad \leq c\left(M^{-2 q}+(\beta N)^{1-r}\right) \mathbb{B}_{\beta, \Omega}^{q, r}\left(\partial_{t} W(t)\right)+\left\|\tilde{W}_{M, N}(t)\right\|_{\Omega}^{2}, \\
& 2\left|G_{2}\left(t, \tilde{W}_{M, N}(t)\right)\right| \leq\left\|v \partial_{x}\left(W(t)-W_{M, N}(t)\right)\right\|_{\Omega}^{2}+\left\|\tilde{W}_{M, N}(t)\right\|_{\Omega}^{2} \\
& \leq c\left(M^{-2 q}+(\beta N)^{1-r}\right) \mathbb{B}_{\beta, \Omega}^{q, r}\left(\partial_{x} W(t)\right)+\left\|\tilde{W}_{M, N}(t)\right\|_{\Omega}^{2} .
\end{aligned}
$$

Let $c_{*}=\max \left\{\frac{\beta_{0}}{\mu}, \beta_{0} \mu, 1\right\}$. We can prove in the same manner that

$$
\begin{aligned}
2 \mid G_{3}(t, & \left.\tilde{W}_{M, N}(t)\right)+G_{4}\left(t, \tilde{W}_{M, N}(t)\right)+G_{5}\left(t, \tilde{W}_{M, N}(t)\right) \mid \\
\leq & c c_{*}\left(M^{-2 q}+(\beta N)^{1-r}\right) \mathbb{B}_{\beta, \Omega}^{q, r}(W(t)) \\
& +\gamma^{2}\left\|\tilde{W}_{M, N}(t)\right\|_{\Omega}^{2}+\frac{1}{2} \beta_{0} \mu\left\|\partial_{v} \tilde{W}_{M, N}(t)\right\|_{\Omega}^{2} .
\end{aligned}
$$


On the other hand, by using (2.29) and (2.30), we observe that if $w_{M, N}(0)=$ ${ }_{*} P_{M, N, \beta, \Omega} W_{0}$, then

$$
\begin{aligned}
\left\|\tilde{W}_{M, N}(0)\right\|_{\Omega}^{2} & \leq\|\|_{*} P_{M, N, \beta, \Omega} W_{0}-W_{0}\left\|_{\Omega}^{2}+\right\| W_{0}-P_{M, N, \beta, \Omega}^{1} W_{0} \|_{\Omega} \\
& \leq c\left(M^{-2 q}+(\beta N)^{1-r}\right)\left({ }_{*} \mathbb{A}_{\beta, \Omega}^{q, r-1}\left(W_{0}\right)+\mathbb{B}_{\beta, \Omega}^{q, r}\left(W_{0}\right)\right) .
\end{aligned}
$$

Because of (2.31), the same estimate is valid if $w_{M, N}(0)={ }_{* *} P_{M, N, \beta, \Omega} W_{0}$.

For simplicity of statements, we shall use the notation

$$
E(u(t))=\|u(t)\|_{\Omega}^{2}+\int_{0}^{t}\left(\beta_{0} \mu\left\|\partial_{v} u(\xi)\right\|_{\Omega}^{2}+\left|\left\|\left.v\right|^{\frac{1}{2}} u(\xi)\right\|_{L^{2}\left(\Gamma_{2}\right)}^{2}\right) d \xi .\right.
$$

Let $c_{0}=2+\beta_{0}+\gamma^{2}+\frac{2 \gamma^{2}}{\beta_{0} \mu}$. By inserting (3.10)-(3.12) into (3.9), we obtain that

$$
\begin{aligned}
\frac{d}{d t} E\left(\tilde{W}_{M, N}(t)\right) & \leq c_{0} E\left(\tilde{W}_{M, N}(t)\right)+c\left(M^{-2 q}+(\beta N)^{1-r}\right) \\
& \cdot\left(c_{*} \mathbb{B}_{\beta, \Omega}^{q, r}(W(t))+\mathbb{B}_{\beta, \Omega}^{q, r}\left(\partial_{t} W(t)\right)+\mathbb{B}_{\beta, \Omega}^{q, r}\left(\partial_{x} W(t)\right)\right) .
\end{aligned}
$$

By multiplying (3.14) by $e^{-c_{0} t}$, integrating the resulting inequality, and using (3.13), we reach

$$
E\left(\tilde{W}_{M, N}(t)\right) \leq c e^{c_{0} t}\left(M^{-2 q}+(\beta N)^{1-r}\right) D_{\beta, \Omega}^{q, r}(W(t)),
$$

where

$$
\begin{gathered}
D_{\beta, \Omega}^{q, r}(W(t))=\int_{0}^{t} e^{-c_{0} \xi}\left(c_{*} \mathbb{B}_{\beta, \Omega}^{q, r}(W(\xi))+\mathbb{B}_{\beta, \Omega}^{q, r}\left(\partial_{\xi} W(\xi)\right)+\mathbb{B}_{\beta, \Omega}^{q, r}\left(\partial_{x} W(\xi)\right)\right) d \xi \\
+{ }_{*} \mathbb{A}_{\beta, \Omega}^{q, r-1}\left(W_{0}\right)+\mathbb{B}_{\beta, \Omega}^{q, r}\left(W_{0}\right) .
\end{gathered}
$$

Finally, a combination of (2.29), (2.30) and (3.15), for $0 \leq t \leq T$, leads to

$$
\left\|W(t)-w_{M, N}(t)\right\|_{\Omega}^{2}+\beta_{0} \mu \int_{0}^{t}\left\|\partial_{v}\left(W(\xi)-w_{M, N}(\xi)\right)\right\|_{\Omega}^{2} d \xi
$$

$$
\leq c\left(M^{-2 q}+(\beta N)^{1-r}\right)\left(e^{c_{0} t} D_{\beta, \Omega}^{q, r}(W(t))+\mathbb{B}_{\beta, \Omega}^{q, r}(W(t))+\beta_{0} \mu \int_{0}^{t} \mathbb{B}_{\beta, \Omega}^{q, r}(W(\xi)) d \xi\right),
$$

provided that $D_{\beta, \Omega}^{q, r}(W(t))$ and $\mathbb{B}_{\beta, \Omega}^{q, r}(W(t))$ are finite.

We may take $w_{M, N}(0)=P_{M, N, \beta, \Omega} W_{0}$ in (3.3). But, in this case, $w_{M, N}(0)$ is not in $V_{M, N, \beta}(\Omega)$. Following the same line as before and using (2.28), we still derive an error estimate like (3.16). But the quantity ${ }_{*} \mathbb{A}_{\beta, \Omega}^{q, r-1}\left(W_{0}\right)$ involved in the estimate is now replaced by $\mathbb{A}_{\beta, \Omega}^{q, r-1}\left(W_{0}\right)$.

3.3. Implementation. In this subsection, we describe the implementation for scheme (3.3) with a nonhomogeneous term $f(x, v, t)$ at its right side. We use the Crank-Nicolson discretization in time $t$, with the mesh size $\tau$.

For simplicity of statements, we use the notation

$$
a_{\Omega}(z, \phi)=\left(v \partial_{x} z, \phi\right)_{\Omega}+\beta_{0}\left(v z, \partial_{v} \phi\right)_{\Omega}+\gamma\left(x \partial_{v} z, \phi\right)_{\Omega}+\beta_{0} \mu\left(\partial_{v} z, \partial_{v} \phi\right)_{\Omega} .
$$

The fully discrete scheme is as follows:

$$
\left\{\begin{array}{c}
\frac{1}{\tau}\left(w_{M, N}(t+\tau)-w_{M, N}(t), \phi\right)_{\Omega}+\frac{1}{2} a_{\Omega}\left(w_{M, N}(t+\tau)+w_{M, N}(t), \phi\right) \\
=\frac{1}{2}(f(t+\tau)+f(t), \phi)_{\Omega}, \\
w_{M, N}(0)={ }_{* *} P_{M, N, \beta, \Omega} W_{0} .
\end{array}\right.
$$


Let

$$
A_{\Omega}(z, u)=\frac{1}{2} \tau a_{\Omega}(z, u)+(z, u)_{\Omega}, \quad G_{\Omega}(z, u)=-\frac{1}{2} \tau a_{\Omega}(z, u)+(z, u)_{\Omega} .
$$

Then, at each time step, we need to solve the equation

$A_{\Omega}\left(w_{M, N}(t), \phi\right)=G_{\Omega}\left(w_{M, N}(t-\tau), \phi\right)+\frac{1}{2} \tau(f(t)+f(t-\tau), \phi)_{\Omega}, \quad \forall \phi \in V_{M, N, \beta}(\Omega)$.

For convenience, we denote $\tilde{\mathcal{L}}_{l}^{(0, \beta)}(v)$ by $\tilde{\mathcal{L}}_{l}^{(\beta)}(v)$. Let

$\psi_{1, l}^{(\beta)}(v)=\tilde{\mathcal{L}}_{l}^{(\beta)}(v)-\tilde{\mathcal{L}}_{l+1}^{(\beta)}(v), \psi_{2, l}^{(\beta)}(v)=\tilde{\mathcal{L}}_{l}^{(\beta)}(-v)-\tilde{\mathcal{L}}_{l+1}^{(\beta)}(-v), \quad 0 \leq l \leq N-1$,

$\eta_{1, m}(x)=L_{m}(x)+L_{m+1}(x), \eta_{2, m}(x)=L_{m}(x)-L_{m+1}(x), \quad 0 \leq m \leq M-1$,

and

$G_{k, l}^{(1, \beta)}(x, v)=\left\{\begin{array}{ll}\eta_{1, k}(x) \psi_{1, l}^{(\beta)}(v), & \text { in } \Omega_{1}, \\ 0, & \text { in } \Omega_{2},\end{array} G_{k, l}^{(2, \beta)}(x, v)= \begin{cases}0, & \text { in } \Omega_{1}, \\ \eta_{2, k}(x) \psi_{2, l}^{(\beta)}(v), & \text { in } \Omega_{2} .\end{cases}\right.$

Obviously, $G_{k, l}^{(1, \beta)}(x, v), G_{k, l}^{(2, \beta)}(x, v) \in V_{M, N, \beta}(\Omega)$. Furthermore, let

$$
\tilde{G}_{m}^{(\beta)}(x, v)=\left(L_{m}(x)-L_{m+2}(x)\right) e^{-\frac{1}{2} \beta|v|} \in V_{M, N, \beta}(\Omega), 0 \leq m \leq M-2 .
$$

The functions $G_{k, l}^{(j, \beta)}(x, v), 0 \leq k \leq M-1,0 \leq l \leq N-1, j=1,2$ and $\tilde{G}_{m}^{(\beta)}(x, v), 0 \leq m \leq M-2$, form a basis of $V_{M, N, \beta}(\Omega)$.

In actual computation, we expand the numerical solution as

$$
\begin{gathered}
w_{M, N}(x, v, t)=\sum_{k=0}^{M-1} \sum_{l=0}^{N-1} v_{k, l}^{(1)} G_{k, l}^{(1, \beta)}(x, v)+\sum_{k=0}^{M-1} \sum_{l=0}^{N-1} v_{k, l}^{(2)} G_{k, l}^{(2, \beta)}(x, v) \\
+\sum_{m=0}^{M-2} v_{m}^{(0)}(t) \tilde{G}_{m}^{(\beta)}(x, v) .
\end{gathered}
$$

Inserting the above expansion into (3.18) and taking $\phi=\tilde{G}_{m^{\prime}}^{(\beta)}(x, v)$ and $\phi=$ $G_{k^{\prime}, l^{\prime}}^{(j, \beta)}(x, v)$, respectively, we derive a matrix form of (3.3), in which all matrices are the tensor products of some tridiagonal symmetric matrices, tridiagonal antisymmetric matrices, upper-triangular matrices and pentadiagonal symmetric matrices. This feature simplifies the calculation. Indeed, this is another advantage of the proposed algorithm.

3.4. Numerical results. We use scheme (3.17) to solve (1.1) with $\beta_{0}=\gamma=\mu=1$ and a non-homogeneous source term $f(x, v, t)$ at the right side. We take the test function:

$$
W(x, v, t)=2^{\frac{1}{2}}(t+2)^{-\frac{1}{2}}\left(1-x^{2} e^{-\frac{1}{10}(t+1)\left(x|v| v+v^{2}\right)}\right) e^{-\frac{1}{2} v^{2}} .
$$

This function has a jump at $v=0$, but

$$
W \in C^{\infty}\left(0, T ; C^{1}(\Omega) \cap H^{2}(\Omega)\right) \subset L^{\infty}\left(0, T ; L^{2}(\Omega)\right) \cap L^{2}(0, T ; \mathbb{M}(\Omega)) .
$$

By this fact and the construction of $w_{M, N}(x, y, t)$, the left side of (3.16) is meaningful. Further, the upper-bound of the numerical solution (cf. the right side of (3.16)) depends on the quantities $D_{\beta, \Omega}^{q, r}(W(t)), \mathbb{B}_{\beta, \Omega}^{q, r}(W(t))$, and so on, which are in turn some combinations of the related norms of $W$, defined on the two subdomains. Since the above test function has a discontinuity only at $v=0$ and is 

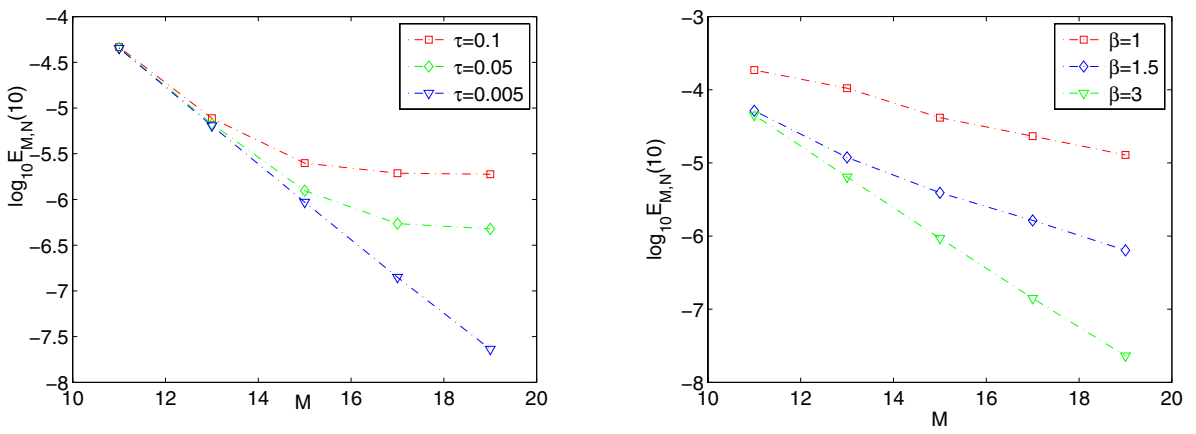

Figure 1. The errors $\log _{10} E_{M, N, \Omega}(10)$. Figure 2. The errors $\log _{10} E_{M, N, \Omega}(10)$.

infinitely continuous on the two subdomains, these quantities are finite for any integers $r>1, q>0$. Accordingly, the numerical solution possesses the numerical accuracy of the order $M^{-q}+N^{\frac{1-r}{2}}$. This implies the spectral accuracy in space, which is conformed by the results stated below; see Figures 1 and 2. It is noted that if $\partial_{v}^{2} W$ has a discontinuity at $v \neq 0$, then a calculation with Remark 2.1 and (3.16) shows that the numerical accuracy is of the order $M^{-q}+N^{-1}$ at least.

Let $x_{M, l}^{(1)}$ and $x_{M, l}^{(2)}$ be the zeros of the polynomials $L_{M}(x)+L_{M+1}(x)$ and $L_{M}(x)-$ $L_{M+1}(x)$, respectively. $\rho_{M, l}^{(1)}$ and $\rho_{M, l}^{(2)}$ stand for the Christoffel numbers of the corresponding Legendre interpolations. Meanwhile, $v_{N, k}^{(1, \beta)}$ and $v_{N, k}^{(2, \beta)}$ are the roots of the polynomials $\mathcal{L}_{N+1}^{(0, \beta)}(v)$ and $\mathcal{L}_{N+1}^{(0, \beta)}(-v)$, respectively. $\omega_{N, k}^{(1, \beta)}$ and $\omega_{N, k}^{(2, \beta)}$ are the Christoffel numbers of the corresponding generalized Laguerre interpolations. Furthermore, let $\tilde{\omega}_{N, k}^{(1, \beta)}=e^{\beta v_{N, k}^{(1, \beta)}} \omega_{N, k}^{(1, \beta)}, \tilde{\omega}_{N, k}^{(2, \beta)}=e^{-\beta v_{N, k}^{(2, \beta)}} \omega_{N, k}^{(2, \beta)}$, and

$$
\|u\|_{M, N}=\left(\sum_{j=1}^{2} \sum_{l=0}^{M} \sum_{k=0}^{N} u^{2}\left(x_{M, l}^{(j)}, v_{N, k}^{(j, \beta)}\right) \rho_{M, l}^{(j)} \tilde{\omega}_{N, k}^{(j, \beta)}\right)^{\frac{1}{2}} .
$$

The numerical errors are measured by the following discrete norm:

$$
E_{M, N}(t)=\left\|W(t)-w_{M, N}(t)\right\|_{M, N} \approx\left\|W(t)-w_{M, N}(t)\right\|_{L^{2}(\Omega)} .
$$

In Figure 1, we plot the errors $\log _{10} E_{M, N}(t)$ with $N=5 M, t=10$ and $\beta=3$. Clearly, the errors decay fast when $M$ and $N$ increase and $\tau$ decreases. It is seen that for the fixed time step size $\tau=0.1$ and the small mode $M \leq 17$, the total numerical errors are dominated by the approximation errors in the space, and so they decay fast as $M$ increases. But for $M \geq 17$, the total numerical errors are dominated by the approximation errors in time $t$. Thus, the numerical results keep the same accuracy, even if $M$ and $N$ increase again. A similar situation happens for $\tau=0.05$. However, for small $\tau \leq 0.005$, the total numerical errors are dominated by the approximation errors in the space, and so they decay very fast as $M$ and $N$ increase. The above facts coincide very well with the theoretical analysis in Subsection 3.3. In particular, they show the spectral accuracy in the space of scheme (3.3).

In Figure 2, we plot $\log _{10} E_{M, N}(t)$ at $t=10$, with $N=5 M, \tau=0.005$ and different values of the parameter $\beta$. It seems that the errors with suitably larger $\beta$ 


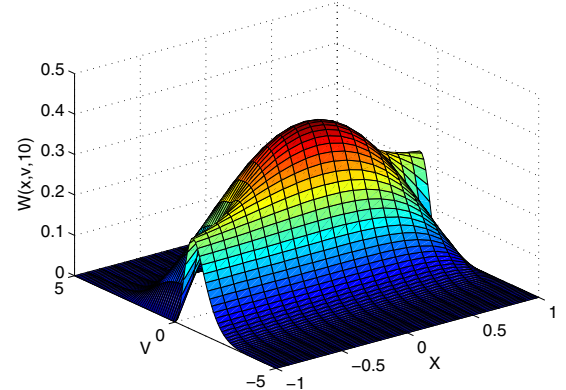

Figure 3. The exact solution, $t=10$.

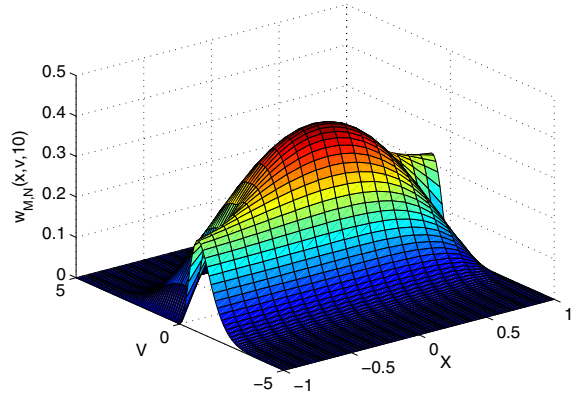

Figure 4. The numerical solution, $t=10$.

are smaller than those with smaller $\beta$. However, how to choose the best parameter $\beta$ is still an open problem. Roughly speaking, if the exact solution decays faster as $|v|$ increases, then it is better to take $\beta$ larger.

In Figure 3 and Figure 4, we plot the exact solution $W(x, v, t)$ and the numerical solution $w_{M, N}(x, v, t)$ with $M=19, N=95, \tau=0.005$ and $\beta=3$, respectively. They demonstrate that the numerical solution fits the exact solution very well.

\section{Concluding Remarks}

In this paper, we proposed the composite generalized Laguerre-Legendre spectral method for the Fokker-Planck equation in an infinite channel, which plays an important role in many fields. The numerical results demonstrated the spectral accuracy in space and coincide well with the theoretical analysis.

The main advantages of the proposed approach are as follows:

- With the aid of composite generalized Laguerre-Legendre approximations, we could deal with PDEs properly, which are of different types on different subdomains. This trick also simplifies actual computations, especially for large modes $M$ and $N$.

- Using the mixed Laguerre-Legendre approximations, we could reasonably approximate those partial differential equations that behave like parabolic equations in one direction and behave like hyperbolic equations in the other direction.

- By using different generalized Laguerre approximations on different subdomains, we could exactly match the singularities of coefficients appearing in the underlying differential equations, which degenerate and grow up in different ways on different subdomains. Consequently, we could deal with the problems on the whole domain properly.

- The adjustable parameter $\beta$ involved in the generalized Laguerre approximation enables us to fit the asymptotic behaviors of exact solutions at infinity closely.

- By using different Legendre approximations on different subdomains, we could fit different kinds of boundary conditions, as imposed in the underlying problems.

Although we only considered the Fokker-Planck equation, the main idea and techniques developed in this paper are also applicable to many other problems of non-standard types. In particular, the results on some quasi-orthogonal approximations are very appropriate for various spectral and pseudospectral methods with 
domain decompositions, as well as nonlinear problems and exterior problems; e.g., see [20, 30].

\section{APPENDIX}

We can follow the same line as in 26] to prove the existence, uniqueness and regularity of the solution of (3.1). We give the sketch of the proof as follows.

Step $I$. Let $\mathbb{M}_{L}$ be a family of finite-dimensional spaces, which approximates the space $\mathbb{M}$ consistently as $L \longrightarrow \infty$. We consider the auxiliary problem

$(A 1)$

$$
\left\{\begin{array}{l}
\left(\partial_{t} W_{L}(t), \phi\right)_{\Omega}-\left(v W_{L}(t), \partial_{x} \phi\right)_{\Omega}+\beta_{0}\left(v W_{L}(t), \partial_{v} \phi\right)_{\Omega}+\gamma\left(x \partial_{v} W_{L}(t), \phi\right)_{\Omega} \\
+\beta_{0} \mu\left(\partial_{v} W_{L}(t), \partial_{v} \phi\right)_{\Omega}+\int_{\Gamma_{2}}|v| W_{L}(\cdot, v, t) \phi(\cdot, v) d v=0, \quad \forall \phi \in \mathbb{M}_{L}, 0<t \leq T \\
W_{L}(0)=W_{L, 0},
\end{array}\right.
$$

where $\left\|W_{L, 0}-W_{0}\right\|_{L_{v^{2}+1}^{2}(\Omega)} \longrightarrow 0$ as $L \longrightarrow \infty$.

Taking $\phi=2 W_{L}$ in $(A 1)$, we obtain that

$$
\partial_{t}\left\|W_{L}(t)\right\|\left\|_{\Omega}^{2}+2 \beta_{0} \mu\right\| \partial_{v} W_{L}(t)\left\|_{\Omega}^{2}+\right\| W_{L}(t)\left\|_{L_{|v|}^{2}\left(\Gamma_{2}\right)}^{2}=\beta_{0}\right\| W_{L}(t) \|_{L^{2}(\Omega)}^{2},
$$

whence

$$
\begin{aligned}
& \left\|W_{L}(t)\right\|_{\Omega}^{2}+2 \beta_{0} \mu \int_{0}^{t}\left\|\partial_{v} W_{L}(\xi)\right\|_{\Omega}^{2} d \xi+\int_{0}^{t}\left\|W_{L}(\xi)\right\|_{L_{|v|}^{2}\left(\Gamma_{2}\right)}^{2} d \xi \\
& =\beta_{0} \int_{0}^{t}\left\|W_{L}(t)\right\|_{L^{2}(\Omega)}^{2} d \xi+\left\|W_{L, 0}\right\|_{\Omega}^{2} \leq \beta_{0} \int_{0}^{t}\left\|W_{L}(t)\right\|_{L^{2}(\Omega)}^{2} d \xi+c\left\|W_{0}\right\|_{L^{2}(\Omega)}^{2} .
\end{aligned}
$$

By the Gronwall inequality, $W_{L}, \partial_{v} W_{L}$ and $\left.W_{L}\right|_{\Gamma_{2}}$ remain in some bounded sets of $L^{\infty}\left(0, T ; L^{2}(\Omega)\right), L^{2}\left(0, T ; L^{2}(\Omega)\right)$ and $L^{2}\left(0, T ; L_{|v|}^{2}\left(\Gamma_{2}\right)\right)$, respectively. Accordingly, we can extract a subsequence, still denoted by $W_{L}$, such that for certain $W^{*}$,

$$
\begin{array}{lll}
W_{L} \longrightarrow W^{*} & \text { in } & L^{2}\left(0, T ; L^{2}(\Omega)\right) \text { weak, } \\
\partial_{v} W_{L} \longrightarrow \partial_{v} W^{*} & \text { in } & L^{2}\left(0, T ; L^{2}(\Omega)\right) \quad \text { weak, } \\
W_{L} \longrightarrow W^{*} & \text { in } & L^{\infty}\left(0, T ; L^{2}(\Omega)\right) \quad \text { weak star, } \\
\left.\left.W_{L}\right|_{\Gamma_{2}} \longrightarrow W^{*}\right|_{\Gamma_{2}} & \text { in } & L^{2}\left(0, T ; L_{|v|}^{2}\left(\Gamma_{2}\right)\right) \quad \text { weak, } \\
\partial_{t} W_{L} \longrightarrow \partial_{t} W^{*} & \text { in } & L^{2}\left(0, T ; L^{2}(\Omega)\right) \quad \text { weak. }
\end{array}
$$

Then by a standard compactness argument, we know that (3.1) has a solution $W$ such that

$(A 2)$

$W \in L^{\infty}\left(0, T ; L^{2}(\Omega)\right), \partial_{v} W \in L^{2}\left(0, T ; L^{2}(\Omega)\right),\left.W\right|_{\Gamma_{1}}=0,\left.W\right|_{\Gamma_{2}} \in L^{2}\left(0, T ; L_{|v|}^{2}\left(\Gamma_{2}\right)\right)$.

Step $I I$. In the sense of distributions, the solution of (3.1) satisfies (A3) $\partial_{t} W+v \partial_{x} W-\beta_{0} \partial_{v}(v W)+\gamma x \partial_{v} W-\beta_{0} \mu \partial_{v}^{2} W=0, \quad(x, v) \in \Omega, 0<t \leq T$.

Following the same line as in [26, we multiply (A3) by $2 v^{2} W$ and integrate the result by parts to derive that

$$
\begin{aligned}
\partial_{t}\|W(t)\|_{L_{v^{2}}^{2}(\Omega)}^{2} & +\beta_{0}\|W(t)\|_{L_{v^{2}}^{2}(\Omega)}^{2}+\|W(t)\|_{L_{|v|^{3}}^{2}\left(\Gamma_{2}\right)}^{2}+2 \beta_{0} \mu\left\|\partial_{v} W(t)\right\|_{L_{v^{2}}^{2}(\Omega)}^{2} \\
& =-2 \gamma \int_{\Omega} x v^{2} W(t) \partial_{v} W(t) d x d v-4 \beta_{0} \mu \int_{\Omega} v W(t) \partial_{v} W(t) d x d v \\
& \leq \beta_{0} \mu\left\|\partial_{v} W(t)\right\|_{L_{v^{2}}^{2}(\Omega)}^{2}+\frac{2 \gamma^{2}}{\beta_{0} \mu}\|W(t)\|_{L_{v^{2}}^{2}(\Omega)}^{2}+8 \beta_{0} \mu\|W(t)\|_{L^{2}(\Omega)}^{2} .
\end{aligned}
$$


Integrating the above inequality with respect to $t$, we obtain that

$$
\begin{aligned}
& \|W(t)\|_{L_{v^{2}}^{2}(\Omega)}^{2}+\beta_{0} \mu \int_{0}^{t}\left\|\partial_{v} W(\xi)\right\|_{L_{v^{2}}^{2}(\Omega)}^{2} d \xi+\int_{0}^{t}\|W(\xi)\|_{L_{|v|}^{2}\left(\Gamma_{2}\right)}^{2} d \xi \\
& \leq\left|\frac{2 \gamma^{2}}{\beta_{0} \mu}-\beta_{0}\right| \int_{0}^{t}\|W(\xi)\|_{L_{v^{2}}^{2}(\Omega)}^{2} d \xi+8 \beta_{0} \mu \int_{0}^{t}\|W(\xi)\|_{L^{2}(\Omega)}^{2} d \xi+\left\|W_{0}\right\|_{L_{v^{2}}^{2}(\Omega)}^{2} .
\end{aligned}
$$

By $(A 2)$, the last two terms of the above inequality are bounded. Therefore, we use the Gronwall inequality to conclude that

$(A 4)$

$$
W \in L^{\infty}\left(0, T ; L_{v^{2}}^{2}(\Omega)\right), \quad \partial_{v} W \in L^{2}\left(0, T ; L_{v^{2}}^{2}(\Omega)\right),\left.\quad W\right|_{\Gamma_{2}} \in L^{2}\left(0, T ; L_{|v|^{3}}^{2}\left(\Gamma_{2}\right)\right) .
$$

The previous statements imply that (3.1) has at least a solution in $L^{\infty}\left(0, T ; L^{2}(\Omega)\right) \cap$ $L^{2}(0, T ; \tilde{\mathbb{M}}(\Omega))$. The uniqueness of the solution is clear. Since $(3.1)$ is a linear problem, we can verify that if $W_{0}$ is smoother, then the solution is also smoother.

\section{REFERENCES}

1. Bernardi C. and Maday Y., Spectral methods, in Handbook of Numerical Analysis, 209-486, ed. by Ciarlet P. G. and Lions J. L., Elsevier, Amsterdam 1997. MR1470226

2. Boyd J. P., Chebyshev and Fourier Spectral Methods, 2nd edition, Dover Publications, Inc., Mineola, New York, 2001. MR1874071 (2002k:65160)

3. Chandrasekhar S., Stochastic problems in physics and astronomy, Rev. Mod. Phys., 15(1934), 1-89. MR0008130 (4:248i)

4. Cartling B., Kinetics of activated processes from nonstationary solutions of the Fokker-Planck equation for a bistable potential, J. Chem. Phys., 87(1987), 2638-2648.

5. Coulaud O., Funaro D. and Kavian O., Laguerre spectral approximation of elliptic problems in exterior domains, Comput. Meth. in Appl. Mech. Engrg., 80 (1990), 451-458. MR1067965

6. Canuto C., Hussaini M. Y., Quarteroni A. and Zang T. A., Spectral Methods in Fluid Dynamics, Springer-Verlag, Berlin, 1988. MR917480 (89m:76004)

7. Diperna R. J. and Lions P. L., On the Fokker-Planck-Boltzmann equation, Commun. Math. Phys., 120(1988), 1-23. MR972541 (90b:35203)

8. Fok J. C. M., Guo Ben-Yu and Tang Tao, Combined Hermite spectral-finite difference method for The Fokker-Planck equation, Math. Comp., 71(2001), 1497-1528. MR.1933042 (2003j:65096)

9. Funaro D., Polynomial Approximations of Differential Equations, Springer-Verlag, Berlin, 1992. MR 1176949 (94c:65078)

10. Funaro D. and Kavian O., Approximation of some diffusion evolution equations in unbounded domains by Hermite functions, Math. Comp., 57(1999), 597-619. MR1094949 (92k:35156)

11. Gottlieb D. and Orszag S. A., Numerical Analysis of Spectral Methods: Theory and Applications, SIAM-CBMS, Philadelphia, 1977. MR0520152 (58:24983)

12. Guo Ben-yu, Spectral Methods and Their Applications, World Scientific, Singapore, 1998. MR.1641586 (2000b:65194)

13. Guo Ben-yu, Error estimation of Hermite spectral method for nonlinear partial differential equations, Math. Comp., 68(1999), 1069-1078. MR.1627789 (99i:65111)

14. Guo Ben-yu, Jacobi approximations in certain Hilbert spaces and their applications to singular differential equations, J. Math. Anal. Appl., 243(2000), 373-408. MR.1741531 (2001b:65082)

15. Guo Ben-yu and Ma He-ping, Composite Legendre-Laguerre approximation in unbounded domains, J. Comput. Math., 19(2001), 101-112. MR1807108(2002b:41005)

16. Guo Ben-yu and Shen Jie, Laguerre-Galerkin method for nonlinear partial differential equations on a semi-infinite interval, Numer. Math., 86(2000), 635-654. MR 1794346|(2001h:65152)

17. Guo Ben-yu, Shen Jie and Wang Li-lian, Optical spectral-Galerkin methods using generalized Jacobi polynomials, J. Sci. Comp., 27(2006), 305-322. MR2285783

18. Guo Ben-yu, Shen Jie and Xu Cheng-Long, Generalized Laguerre approximation and its applications to exterior problems, J. Comput. Math., 23(2005), 113-130. MR2118049 (2005m:65289)

19. Guo Ben-yu and Wang Li-lian, Jacobi approximations in non-uniformly Jacobi-weighted Sobolev spaces, J. Appr. Theory, 128(2004), 1-41. MR2063010(2005h:41010) 
20. Guo Ben-yu and Wang Tian-jun, Composite Laguerre-Legendre spectral method for exterior problems, submitted.

21. Guo Ben-yu and Xu Cheng-long, Mixed Laguerre-Legendre pseudospectral method for incompressible fluid flow in an infinite strip. Math. Comp., 73(2004), 95-125. MR.2034112 (2004m:65157)

22. Guo Ben-yu and Zhang Xiao-yong, A new generalized Laguerre approximation and its applications, J. Comp. Appl. Math., 181(2005), 342-363. MR2146844(2006e:65180)

23. Guo Ben-yu and Zhang Xiao-yong, Spectral method for differential equations of degenerate type on unbounded domains by using generalized Laguerre functions, Appl. Numer. Math., 57(2007), 455-471. MR2310760

24. Maday Y., Pernaud-Thomas B. and Vandeven H., Une réhabilitation des méthods spèctrales de type Laguerre, Rech. Aérospat., 6(1985), 353-375. MR.850680(88b:65135)

25. Moore P. and Flaherty J., Adaptive local overlapping grid methods for parabolic systems in two space dimensions, J. Comp. Phys., 98(1992), 54-63.

26. Perthame B., Higher moments for kinetic equations, The Vlasov-Poisson and Fokker-Planck cases, Math. Meth. Appl. Sci., 13(1990), 441-452. MR.1078593(91j:82044)

27. Risken H., The Fokker-Planck equation: Methods of solution and applications, 2nd ed., Springer-Verlag, Berlin, 1989. MR987631 (90a:82002)

28. Shen Jie, Stable and efficient spectral methods in unbounded domains using Laguerre functions, SIAM J. Numer. Anal., 38(2000), 1113-1133. MR1786133(2001g:65165)

29. Tang T., Mckee S. and Reeks M. W., A spectral method for the numerical solutions of a kinetic equation describing the dispersion of small particles in a turbulent flow, J. Comp. Phys., 103(1991), 222-230.

30. Wang Tian-jun and Guo Ben-yu, Composite Laguerre-Legendre pseudospectral method for Fokker-Planck equation in an infinite strip, Appl. Numer. Math., doi:10.1016/ j.apnum.2007.08.007.

31. Xu Cheng-long and Guo Ben-yu, Mixed Laguerre-Legendre spectral method for incompressible flow in an infinite strip, Adv. in Comput. Math., 16(2002), 77-96. MR.1888220(2002m:65103)

32. Xu Cheng-long and Guo Ben-yu, Laguerre pseudospectral method for nonlinear partial differential equations, J. Comput. Math., 20(2002), 413-428. MR1914675 (2003e:65184)

Department of Mathematics, Shanghai Normal University, Shanghai, 200234, China, Scientific Computing Key Laboratory of Shanghai Universities, Division of Computational Science of E-Institute of Shanghai Universities

E-mail address: byguo@shnu.edu.cn

Department of Mathematics and Physics, Henan University of Science and TechnolOGY, LuOYAnG, 471003, China

E-mail address: wangtianjun64@163.com 